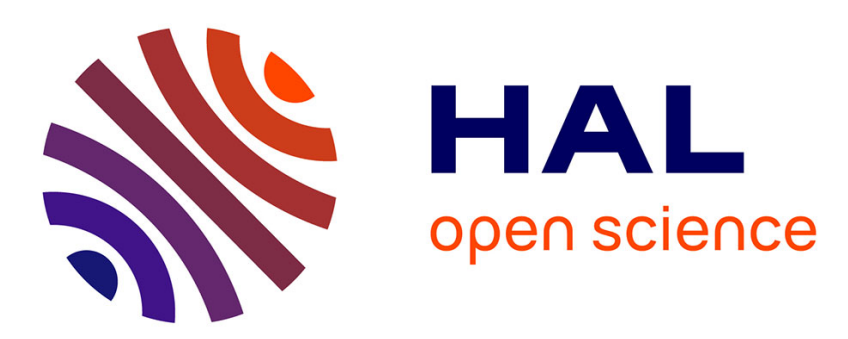

\title{
The reduced model multiscale method (R3M) for the non-linear homogenization of hyperelastic media at finite strains
}

\author{
Julien Yvonnet, Qi-Chang He
}

\section{- To cite this version:}

Julien Yvonnet, Qi-Chang He. The reduced model multiscale method (R3M) for the non-linear homogenization of hyperelastic media at finite strains. Journal of Computational Physics, 2007, 223 (1), pp.341-368. 10.1016/j.jcp.2006.09.019 . hal-00693621

\section{HAL Id: hal-00693621 \\ https://hal.science/hal-00693621}

Submitted on 7 Sep 2014

HAL is a multi-disciplinary open access archive for the deposit and dissemination of scientific research documents, whether they are published or not. The documents may come from teaching and research institutions in France or abroad, or from public or private research centers.
L'archive ouverte pluridisciplinaire HAL, est destinée au dépôt et à la diffusion de documents scientifiques de niveau recherche, publiés ou non, émanant des établissements d'enseignement et de recherche français ou étrangers, des laboratoires publics ou privés. 


\title{
The Reduced Model Multiscale Method (R3M) for the non-linear homogenization of hyperelastic media at finite strains
}

\author{
J. Yvonnet*, Q.-C. He \\ ${ }^{a}$ Université de Marne-la-Vallée, Laboratoire de Mécanique, \\ EA 2545, 5 Bd Descartes, F-77454 Marne-la-Vallée Cedex 2, France
}

\begin{abstract}
This paper presents a new multi-scale method for the homogenization analysis of hyperelastic solids undergoing finite strains. The key contribution is to use an incremental nonlinear homogenisation technique in tandem with a model reduction method, in order to alleviate the complexity of multiscale procedures, which usually involve a large number of nonlinear nested problems to be solved. The problem associated with the Representative Volume Element (RVE) is solved via a model reduction method (Proper Orthogonal Decomposition). The reduced basis is obtained through pre-computations on the RVE. The technique, coined as Reduced Model Multiscale Method (R3M), allows reducing significantly the computation times, as no large matrix needs to be inverted, and as the convergence of both macro and micro problems is enhanced. Furthermore, the R3M drastically reduces the size of the data base describing the history of the micro problems. In order to validate the technique in the context of porous elastomers at finite strains, a comparison between a full and a reduced multiscale analysis is performed through numerical examples, involving different micro and macro structures, as well as different nonlinear models (Neo-Hookean, Mooney-Rivlin). It is shown that the R3M gives good agreement with the full simulations, at lower computational and data storage requirements.
\end{abstract}

Key words: Model reduction, Proper orthogonal decomposition, Multiscale analysis, Nonlinear homogenization, Finite strains.

\footnotetext{
* Correspondance to J. Yvonnet

Email address: jyvonnet@univ-mlv.fr (J. Yvonnet).
} 


\section{Introduction}

Homogenization of heterogeneous solids in a geometrically and physically nonlinear regime is a challenging problem in computational mechanics $[52,11]$. Furthermore, the question of characterizing the behaviour of heterogeneous media undergoing finite deformations arises in many modern applications, such as biological tissues [24], and reinforced rubbers [56]. Whereas homogenization techniques have been widely used and have proved to be efficient tools in the context of linear heterogeneous materials, most of them are not suitable to deal with large deformations, complex loading paths, and cannot account for an evolving micro-structure. Foundations of the homogenization of heterogeneous materials are outlined in Willis [63], Suquet [59], Müller [45], Nemat-Nasser and Hori [46], Ponte Castañeda and Suquet [52] and Miehe et al. [41], among others. Determining an effective energy-density function for describing the overall behaviour of composites was extended to finite deformation elasticity in the pioneering works of Hill [19], Hill and Rice [20] and Ogden [48].

Analytical approaches are in many circumstances restricted, especially with regard to the geometry of the representative micro-structure and its constitutive response which is often assumed to be linearly elastic. In the context of hyperelastic media, some estimates exist for special loadings [18], as well as other estimates based on various types of ad hoc approximations, mostly for low-density foams (see i.e., $[10,12])$. Bounds on the overall strain energydensity functions of geometrically nonlinear composites were determined by Ogden [49] and Ponte Castañeda [51]. More recently, Ponte Castañeda [53] and Lopez-Pamies and Ponte Castañeda [35] developed a variational procedure for determining the effective properties of composites undergoing finite deformations and obtained some specific results for the class of transversely isotropic composites, and generated estimates for effective behaviour and loss of ellipticity in hyperelastic porous materials with random microstructures subjected to finite deformations. deBotton et al. [4] have considered the response of a transversely isotropic fiber-reinforced composite made out of two incompressible neo-Hookean phases undergoing finite deformations. They developed an expression for the effective energy density function of the composites in terms of the volume fractions of the phases.

Modelling heterogeneous materials by meshing the whole structure, including all heterogeneities, leads to giant computations. Such an approach may be practicable for some very specific structures where the heterogeneities are quite big, and where the material is linear. Recently, some attempt have been made to re-formulate this global problem and consequently to try to reduce the computational cost [29].

Alternatively, computational or incremental homogenization techniques have 
been developed, which are essentially based on the solution of two (nested) boundary value problems, one for the macroscopic and one for the microscopic scale. In techniques of this type, e.g. [58,16,61,14,57,41,42,38,8,9,62,15,28], among others, the macroscopic deformation (gradient) tensor is calculated for every material point of the macrostructure and is next used to formulate kinematic boundary conditions to be applied on the associated microstructural representative volume element (RVE). After the solution of the microstructural boundary value problem, the macroscopic stress tensor is obtained by averaging the resulting microstructural stress field over the volume of the microstructural cell. As a result, the (numerical) stress-strain relationship at every macroscopic point is readily available.

Techniques of this type offer the following advantages: (a) large deformations and rotations on both micro and macro level can be incorporated; (b) arbitrary behaviour, including physically non-linear and time-dependant behaviour can be used to model the micro level; (c) detailed microstructural information, including a physical and/or geometrical evolution of the microstructure, can be introduced in the macroscopic analysis; (d) different discretization techniques (finite element, meshfree methods, boundary element methods...) can be used at both levels.

Most of these techniques are called first-order, in which the assumption that the microstructural length scale is infinitely small compared to the characteristic macro structural size. Second-order homogenization have been proposed by Kouznetsova et al. [28] to handle problems where both length scales become comparable, or when highly localized deformations occur. A similar approach have been proposed by Feyel in [9]. Direct micro-to-macro transitions based on finite element formulations of inelastic heterogeneous materials in the largestrains context have recently been considered for example by Smit et al. [57], Miehe et al. [41], Kouznetsova et al. [27] and Miehe et al. [44].

Despite the cost is far less expensive than the brute force approach, these techniques still lead to large computations, as many non-linear problems have to be solved, while the data necessary for the incremental resolution have to be stored for each problem, generating a large database. One solution is the use of parallel computations [8].

Alternatively, model reduction can significantly reduce time and data storage requirements. In [39,40], Michel and Suquet proposed an approximate model for describing the overall hardening of elastoplastic or elastoviscoplastic composites using non uniform transformation fields, generalizing the idea of Dvorak [6]. This analysis delivers effective constitutive relations for nonlinear composites expressed in terms of a reduced number of internal variables which are the components of the microscopic plastic field over a finite set of plastic modes. In the mentioned work, the plastic modes were chosen as actual 
plastic fields in the composite under some specific loadings.

The proper orthogonal decomposition (POD), is a powerful and elegant method for data analysis, aimed at obtaining low-dimensional approximate descriptions of a higher-dimensional process. The POD provides a basis for the modal decomposition of an ensemble of functions, such as data obtained in the course of experiments or numerical simulations. The most striking features of the POD is its optimality: it provides the most efficient way of capturing the dominant components of an infinite-dimensional process with only a finite number of modes, often surprisingly few modes [3,22]. The technique seems adapted to the aforementioned multiscale approaches, where numerous nonlinear problems have to be solved repeatedly.

The central contribution of this paper is the development of a reduced model multiscale method (R3M), proposed for homogenization of nonlinear hyperelastic problems at finite strains. In the context of R3M, a reduced model substitutes the full problem describing the nonlinear micro problem. The reduced basis is obtained through a POD procedure. For this purpose, precomputations are performed on the RVE subjected to different applied loads. The main aim of this work is to evaluate the capabilities of the method in the context of non-linear hyperelastic problems at finite strains and to compare it with a full computation. For sake of simplicity, we will focus on problems where no loss of ellipticity occurs.

The layout of this paper is as follows. In section 2, an overview of Proper Orthogonal Decomposition is provided. In section 3, the boundary-value problem associated with non-homogeneous hyperelastic material is formulated. In section 4, the reduced model multiscale method (R3M) is presented. Finally, the R3M is evaluated through different numerical examples in section 5 .

\section{Model reduction using the proper orthogonal decomposition}

The proper orthogonal decomposition [37] is obtained by a procedure which goes back at least to the papers of Pearson [50] and Schmidt [54], and which reappears under a multitude of names, such as the Karhunen-Loève transform (KLT) [26,34], principal component analysis [21], proper orthogonal eigenfunctions [36], factor analysis [17], and total least squares [13]. The singular value decomposition algorithm [13] is a key to the understanding of these methods.

The POD was initially designed to analyze random process data by introducing new coordinate systems based on its statistical properties. It does not only provide structures within random data, but also leads to more efficient way of coordinate description. These characteristics make the POD a suitable 
tool for various tasks ranging from data analysis and compression to model order reduction. The POD identifies a useful set of basis functions and the dimension of the subspace necessary to achieve a satisfactory approximation of the system. The POD also facilitates the resolution of the partial differential equations through their projection into a reduced-order model [1].

Applications of this approach are found in many engineering and scientific disciplines, such as random variables analysis, image processing, signal analysis, data compression, process identification and control in chemical engineering, oceanography, etc. [22]. The POD has been used to obtain approximate, lowdimensional descriptions of turbulent fluid flows [22,37], structural vibrations and chaotic dynamical systems [7]. Many recent investigations have examined impacting systems $[1,2]$ and thermics [47].

In particular, the KLT best approximates a stochastic process in the least square sense. It can be formulated for both continuous and discrete time. In the following, we focus on the discrete KLT for incremental nonlinear mechanical analysis. The mathematical theory of the KLT relies on the properties of Hilbert spaces. A Hilbert space $H$ is a vector space that is complete as a metric space and has a scalar product $\langle.,$.$\rangle . The norm is defined as \|\boldsymbol{\psi}\|=\sqrt{\langle\boldsymbol{\psi}, \boldsymbol{\psi}\rangle}$ for $\boldsymbol{\psi} \in H$ and the metric is defined as $d(\boldsymbol{\psi}, \boldsymbol{\phi})=\|\boldsymbol{\psi}-\boldsymbol{\phi}\|$ for $\boldsymbol{\psi}, \boldsymbol{\phi} \in H$. Without loss of generality we will consider in his paper Hilbert spaces only in $\Re^{N}$. The concept of orthogonality and orthonormality is crucial for the derivation of the KLT: two vectors $\boldsymbol{\psi}_{i}, \boldsymbol{\psi}_{j} \in H$ are orthonormal if $\left\langle\boldsymbol{\psi}_{i}, \boldsymbol{\psi}_{j}\right\rangle=\delta_{i j}$. A basis $\boldsymbol{\Psi}$ of a Hilbert space is orthonormal if any two distinct vectors $\boldsymbol{\psi}_{i}, \boldsymbol{\psi}_{j} \in \boldsymbol{\Psi}$ are orthogonormal.

We consider a solid $D$-dimensional body subjected to a time-dependent quasistatic loading during a time interval $I=[0, T]$ discretized by $S$ instants $\left\{t_{1}, t_{2}, \ldots, t_{S}\right\}$. Let $\mathbf{q}_{i}$ denote the $D N$-dimensional vector formed by the displacement components of $N$ points of the solid recorded at an instant $t_{i} \in I$.

Next, we consider a time-dependant vector $\mathbf{q}^{R}(t) \in \Re^{D N}$ and the following expansion:

$$
\mathbf{q}^{R}(t)=\phi_{0}+\sum_{m=1}^{M} \phi_{m} \xi_{m}(t)
$$

with $M<D N, \phi_{0}$ and $\phi_{m}(m=1, \ldots, M)$ are constant vectors belonging to $\Re^{D N}$, and $\xi_{m}(t)$ are scalar functions of time $t$. The time dependent vectors $\mathbf{q}^{R}(t)$ given by (1) is required to minimize:

$$
\sum_{i=1}^{S}\left\|\mathbf{q}\left(t_{i}\right)-\mathbf{q}^{R}\left(t_{i}\right)\right\|^{2}
$$


with the constraints:

$$
\left\langle\phi_{i}, \phi_{j}\right\rangle=\delta_{i j}
$$

Solving this constrained optimization problem gives $\phi_{0}$ (see i.e. $[31,5]$ ) as:

$$
\phi_{0}=\overline{\mathbf{q}}=\frac{1}{S} \sum_{i=1}^{S} \mathbf{q}\left(t_{i}\right)
$$

and $\phi_{i}(i=1, \ldots, D N)$ as the eigenvectors of the eigenvalue problem:

$$
\mathrm{Q} \phi_{i}=\lambda_{i} \phi_{i}
$$

Above, $\mathbf{Q}$ is the covariance matrix defined by:

$$
\mathbf{Q}=\mathbf{U U}^{T}
$$

where the matrix $\mathbf{U}$ is a $(D N \times S)$ matrix with the centred vectors as columns:

$$
\mathbf{U}=\left\{\mathbf{q}\left(t_{1}\right)-\overline{\mathbf{q}}, \mathbf{q}\left(t_{2}\right)-\overline{\mathbf{q}}, \ldots, \mathbf{q}\left(t_{S}\right)-\overline{\mathbf{q}}\right\}
$$

Note that $\mathbf{Q}$ is a semi-definite $(D N \times D N)$ matrix, whose eigenvalues $\lambda_{i}$ are decreasingly ordered: $\lambda_{1} \geq \lambda_{2} \geq \ldots \lambda_{M} \geq \ldots \geq \lambda_{D N} \geq 0$.

A reduced model can be obtained by using only a small number $M$ of basis functions in Eq. (1). If $M<D N$, it can be shown (see i.e. [31]) that the error induced by the K-L procedure is given by:

$$
\epsilon(M)=\sum_{i=1}^{S}\left\|\mathbf{q}\left(\mathbf{x}, t_{i}\right)-\mathbf{q}^{R}\left(\mathbf{x}, t_{i}\right)\right\|=\left(\sum_{i=M+1}^{D N} \lambda_{i}\right)^{1 / 2}
$$

where $M$ is the number of selected basis functions.

The number of basis functions $M$ is then chosen such that

$$
\frac{\left(\sum_{i=M+1}^{D N} \lambda_{i}\right)^{1 / 2}}{\left(\sum_{i=1}^{D N} \lambda_{i}\right)^{1 / 2}}<\delta
$$

where $\delta$ is a given tolerance error parameter, small compared to one. 


\section{Formulation of the inhomogeneous hyperelastic material prob- lem}

\subsection{Macro problem}

let $\Omega^{0}$ be the open domain in $\Re^{D}$ that a D-dimensional solid occupies in its reference configuration and let $\partial \Omega_{0}$ denote the boundary of $\Omega^{0}$. The current configuration and the associated boundary of the solid are referred to as $\Omega(t)$ and $\partial \Omega(t)$. We define $\partial \Omega_{0 u}$ and $\partial \Omega_{0 \sigma}$ as the portions of the prescribed displacements and tractions, respectively. We assume that $\partial \Omega_{0}=\partial \Omega_{0 u} \cup \partial \Omega_{0 \sigma}$ and $\partial \Omega_{0 u} \cap \partial \Omega_{0 \sigma}=\oslash$. Let $\mathbf{u}(t) \in H_{1}\left(\Omega_{0}, t\right)$ the macroscopic displacement field for a given instant $t \in I$. The current position vector $\mathbf{x}(t)$ of a particle of the solid at $t$ is related to its reference position $\mathbf{X}$ by:

$$
\mathbf{x}(\mathbf{X}, t)=\mathbf{X}+\mathbf{u}(\mathbf{X}, t)
$$

Let $\overline{\mathbf{F}}=\boldsymbol{\nabla}_{X} \mathbf{u}+\mathbf{1}$ the macroscopic deformation gradient tensor. The macroscopic nominal stresses tensor $\overline{\mathbf{P}}$ is related to $\overline{\mathbf{F}}$ by:

$$
\overline{\mathbf{P}}=\frac{\partial \bar{\Psi}(\overline{\mathbf{F}})}{\partial \overline{\mathbf{F}}}
$$

where $\bar{\Psi}$ represents the strain-energy function describing the homogenized material. At both the micro and macro scales, $\Psi=\Psi(\mathbf{F})$ is assumed to be continuous and satisfy the principle of frame invariance, i.e. $\Psi(\mathbf{Q F})=\Psi(\mathbf{F})$ for all orthogonal tensors $\mathbf{Q}$. Furthermore, the reference configuration is taken to be stress-free, so that $\Psi(\mathbf{1})=0$.

We assume quasi-static deformations of the body over the time interval $I$ (here the time denotes the loading increment, which varies with time). The problem to solve is defined as follow:

$$
\boldsymbol{\nabla} \cdot \overline{\mathbf{P}}+\overline{\mathbf{B}}=0 \text { and } \overline{\mathbf{P}} \overline{\mathbf{F}}^{T}=\left(\overline{\mathbf{P}} \overline{\mathbf{F}}^{T}\right)^{T} \quad \text { in } \Omega_{0}
$$

where $\overline{\mathbf{B}}$ is a body force term. In (12), the second equation is due to the moment equilibrium. The boundary conditions are defined by:

$$
\left\{\begin{array}{lll}
\mathbf{u}(\mathbf{X})=\overline{\mathbf{u}}(\mathbf{X}) & \text { on } & \partial \Omega_{0 u} \\
\overline{\mathbf{P}} \mathbf{N}=\overline{\mathbf{t}} & \text { on } & \partial \Omega_{0 \sigma}
\end{array}\right.
$$

At both the macro and micro scales, $\mathbf{P}$ is related to the Cauchy stress $\boldsymbol{\sigma}$ by $\mathbf{P}=J \boldsymbol{\sigma} \mathbf{F}^{-T}, J=\operatorname{det}(\mathbf{F})$. The weak form associated with the balance equation (12) is given as follows: 
Find $\mathbf{u} \in H^{1}\left(\Omega_{0}\right)$ verifying the boundary conditions $\mathbf{u}=\overline{\mathbf{u}}$ on $\partial \Omega_{0 u}$ such that, $\forall t \in[0, T]:$

$$
\begin{aligned}
& \int_{\Omega_{0}} \overline{\mathbf{P}}(t): \nabla_{X}(\boldsymbol{\delta} \mathbf{u}) d \Omega=\int_{\Omega_{0}} \overline{\mathbf{S}}: \delta \overline{\mathbf{E}} d \Omega= \\
& \int_{\Omega_{0}} \overline{\mathbf{B}} \cdot \boldsymbol{\delta} \mathbf{u} d \Omega+\int_{\partial \Omega_{0 \sigma}} \overline{\mathbf{t}} \cdot \boldsymbol{\delta} \mathbf{u} d \Gamma \quad \forall \boldsymbol{\delta} \mathbf{u} \in H_{0}^{1}\left(\Omega_{0}\right)
\end{aligned}
$$

or in a more compact form:

$$
\delta \bar{W}_{i n t}=\delta \bar{W}_{e x t}
$$

where $H^{1}\left(\Omega_{0}\right)$ and $H_{0}^{1}\left(\Omega_{0}\right)$ are the usual Sobolev spaces. In (14) $\mathbf{S}$ denotes the second Piola Kirchhoff stress tensor, related to $\mathbf{P}$ through $\mathbf{P}=\mathbf{F S}$, and $\boldsymbol{\delta} \mathbf{E}$ is expressed by:

$$
\boldsymbol{\delta} \mathbf{E}=\frac{1}{2}\left[\mathbf{F}^{T} \nabla_{X}(\delta \mathbf{u})+\nabla_{X}(\delta \mathbf{u})^{T} \mathbf{F}\right]
$$

In order to solve the nonlinear problem (14), an incremental procedure is required, e.g. a Newton-Raphson procedure, implying the linearization of (14), which leads to the set of linear increments [23]:

$$
\begin{aligned}
& D_{\Delta \mathbf{u}} \delta W_{i n t}(\mathbf{u}, \delta \mathbf{u})=\int_{\Omega_{0}}\left[\boldsymbol{\nabla}_{X}(\delta \mathbf{u}): \nabla_{X}(\Delta \mathbf{u}) \overline{\mathbf{S}}\right. \\
& \left.+\overline{\mathbf{F}}^{T} \boldsymbol{\nabla}_{X}(\delta \mathbf{u}): \overline{\mathbf{C}}^{e}: \overline{\mathbf{F}}^{T} \boldsymbol{\nabla}_{X}(\Delta \mathbf{u})\right] d \Omega
\end{aligned}
$$

where $\overline{\mathbf{C}}^{e}$ denotes the fourth-order homogenized material elasticity tensor. We note that in the case of inhomogeneous hyperelastic materials, $\bar{\Psi}$ is not known in general. The elasticity tensor $\overline{\mathbf{C}}^{e}$ can thus not be expressed in closed-form. In order to determine the macroscopic stress-strain relationship, we formulate the problem describing structure at the microlevel in the former section.

\subsection{Micro problem}

Let $\Omega_{\mu}^{0}$ be a representative volume element at the micro scale in the neighbourhood of a macro point $\mathbf{X}$ (see figure 1 ). We assume that $\Omega_{\mu}^{0}$ has a characteristic length much smaller than the characteristic dimension of the structure. Following similar definitions from former section, and denoting by $(.)_{\mu}$ the micro quantities, we assume the existence of a strain energy function $\Psi(t)$ such as the microscopic nominal stresses are related to the microscopic gradient of the 
transformation by:

$$
\mathbf{P}=\frac{\partial \Psi(\mathbf{F})}{\partial \mathbf{F}}
$$

with $\mathbf{F}=\boldsymbol{\nabla}_{X} \mathbf{u}_{\mu}+\mathbf{1}$. The weak form associated with the balance equation over $\Omega_{\mu}^{0}$ is defined by:

Find $\mathbf{u}_{\mu}(t) \in H^{1}\left(\Omega_{\mu}^{0}\right)$ satisfying $\mathbf{u}_{\mu}=\overline{\mathbf{u}}_{\mu}$ on $\partial \Omega_{\mu u}^{0}$ such that, $\forall t \in[0, T]$ :

$$
\begin{aligned}
& \int_{\Omega_{\mu}^{0}} \mathbf{P}(t): \nabla_{X}(\boldsymbol{\delta} \mathbf{u}) d \Omega= \\
& \int_{\Omega_{\mu}^{0}} \mathbf{B} \cdot \boldsymbol{\delta} \mathbf{u} d \Omega+\int_{\partial \Omega_{\mu_{\sigma}}^{0}} \mathbf{t} \cdot \boldsymbol{\delta} \mathbf{u} d \Gamma \quad \forall \boldsymbol{\delta} \mathbf{u} \in H_{0}^{1}\left(\Omega_{\mu}^{0}\right)
\end{aligned}
$$

where $\mathbf{B}$ is the local body force term and $\mathbf{t}$ are the applied tractions. To complete the problem, we need to specify some appropriate boundary conditions for the micro problem. This point will be detailed in the next section. At the microscale, we assume that the behaviour of different constituents is known. In this work, we consider a porous material with a hyperelastic model describing the behaviour of the matrix. More precisely, the compressible Mooney-Rivlin model is characterized by the energy function [23]:

$$
\Psi=c(J-1)^{2}-d \log (J)+c_{1}\left(I_{1}-3\right)+c_{2}\left(I_{2}-3\right)
$$

where $I_{1}, I_{2}$ and $J$ are given by:

$$
I_{1}=\operatorname{Tr}(\mathbf{C}) \quad, \quad I_{2}=\frac{1}{2}\left[\operatorname{Tr}(\mathbf{C})^{2}-\operatorname{Tr}\left(\mathbf{C}^{2}\right)\right] \quad, \quad J=\sqrt{\operatorname{det}(\mathbf{C})}
$$

with $\mathbf{C}=\mathbf{F}^{T} \mathbf{F}$ the right Cauchy-Green tensor. In (20) $c, c_{1}$ and $c_{2}$ are material constants and $d$ defines a (dependent) parameter with certain restrictions. By recalling the assumption that the reference configuration is stress-free we may deduce from $(20)$ that $d=2\left(c_{1}+2 c_{2}\right)$. A special case of the strain-energy (20) is found by taking $c_{2}=0$, leading to the compressible neo-Hookean model.

Linearization of (19) is obtained by substituting the macro quantities for the micro ones in (17). The associated stress tensors and elasticity tensors are given in appendix 7.1 (Eq. (46) and (50), respectively), in the special case of the Mooney-Rivlin model (20). The matrix forms obtained through a Galerkin (finite element) discretization, are given in appendix 7.2.

In the micro domain, we assume that the current position of the material points is the superposition of an average field and a fluctuating field $\mathbf{w}\left(\mathbf{X}_{\mu}\right)$ 
induced by the presence of heterogeneities:

$$
\mathbf{x}_{\mu}=\overline{\mathbf{F}} \mathbf{X}_{\mu}+\mathbf{w}\left(\mathbf{X}_{\mu}\right)
$$

we thus have:

$$
\mathbf{F}=\overline{\mathbf{F}}+\nabla_{X} \mathbf{w}\left(\mathbf{X}_{\mu}\right)
$$

\subsection{Coupling between scales}

In the present paper, we aim at solving iteratively the problems (14) in the structure and (19) in each macro integration point. The coupling between the scales is performed in the following way: (a) specific deformation-driven boundary conditions are imposed on the RVE; (b) after solving the micro problem, the macro stress are recovered by an averaging procedure of the micro stresses. An iterative procedure, e.g. Newton-Raphson technique, is then used to satisfy (14) and (19) at every integration point. In order to specify the boundary conditions on the RVE, we note the additional constraint:

$$
\overline{\mathbf{F}}(\mathbf{X})=\frac{1}{\Omega_{\mu}^{0}} \int_{\Omega_{\mu}^{0}} \mathbf{F}\left(\mathbf{X}_{\mu}\right) d \Omega
$$

where $\overline{\mathbf{F}}\left(\mathbf{X}_{\mu}\right)$ denotes the homogenized gradient of the transformation associated with the point of the macrostructure $\mathbf{X}$. Introducing (22) in (24), it reads:

$$
\begin{aligned}
& \overline{\mathbf{F}}(\mathbf{X})=\frac{1}{\Omega_{\mu}^{0}} \int_{\Omega_{\mu}^{0}} \overline{\mathbf{F}}(\mathbf{x}) d \Omega+\frac{1}{\Omega_{\mu}^{0}} \int_{\Omega_{\mu}^{0}} \nabla_{X} \mathbf{w}\left(\mathbf{X}_{\mu}\right) d \Omega \\
& =\overline{\mathbf{F}}(\mathbf{x})+\frac{1}{\Omega_{\mu}^{0}} \int_{\partial \Omega_{\mu}^{0}} \mathbf{w}\left(\mathbf{X}_{\mu}\right) \otimes \mathbf{N} d \Gamma
\end{aligned}
$$

which imposes:

$$
\frac{1}{\Omega_{\mu}^{0}} \int_{\partial \Omega_{\mu}^{0}} \mathbf{w}\left(\mathbf{X}_{\mu}\right) \otimes \mathbf{N} d \Gamma=0
$$

with $\mathbf{N}$ the unit outward normal on $\partial \Omega_{\mu}^{0}$. The condition (26) is satisfied for the following local boundary conditions:

(i) $\quad \mathbf{w}\left(\mathbf{X}_{\mu}\right)=\mathbf{0}$ on $\partial \Omega_{\mu}^{0}$ and $(i i) \quad \mathbf{w}^{+}\left(\mathbf{X}_{\mu}\right)=\mathbf{w}^{-}\left(\mathbf{X}_{\mu}\right)$ on $\partial \Omega_{\mu}^{0}$ 
The first condition (27 (i)) is satisfied by using homogeneous deformations on the boundary

$$
\mathbf{x}_{\mu}=\overline{\mathbf{F}} \mathbf{X}_{\mu} \quad \forall X_{\mu} \in \partial \Omega_{\mu} \quad \text { or } \quad \mathbf{u}_{\mu}=[\overline{\mathbf{F}}-\mathbf{1}] \mathbf{X}_{\mu} \quad \forall X_{\mu} \in \partial \Omega_{\mu}
$$

The second condition (27 (ii)) states a non-trivial periodicity of the superimposed fluctuation $\mathbf{w}$ on $\partial \Omega_{\mu}^{0}$. Here the boundary is understood to be decomposed into two parts $\partial \Omega_{\mu}^{0}=\partial \Omega_{\mu}^{+} \cup \partial \Omega_{\mu}^{-}$with outward normals $\mathbf{N}^{+}=-\mathbf{N}^{-}$ at two associated points $\mathbf{X}_{\mu}^{+} \in \partial \Omega_{\mu}^{+}$and $\mathbf{X}_{\mu}^{-} \in \partial \Omega_{\mu}^{-}$. A third condition can be expressed, associated with homogeneous stress $\mathbf{t}=\overline{\mathbf{P}} \mathbf{N}$ on the boundary $\partial \Omega_{\mu}^{0} \equiv \partial \Omega_{\mu \sigma}^{0}[43]$.

In this work, we consider only the condition (28) for the sake of simplicity.

The macro stresses are recovered through:

$$
\overline{\mathbf{P}}(t)=\frac{1}{\Omega_{\mu}^{0}} \int_{\Omega_{\mu}^{0}} \mathbf{P}\left(\mathbf{X}_{\mu}, t\right) d \Omega
$$

Using the equilibrium of couples acting on the micro-structure [43]:

$$
\int_{\partial \Omega_{\mu}^{0}}\left(\mathbf{t} \otimes \mathbf{x}_{\mu}-\mathbf{x}_{\mu} \otimes \mathbf{t}\right) d \Gamma=\mathbf{0}
$$

and the identity:

$$
\overline{\mathbf{P}}=\frac{1}{\Omega_{\mu}^{0}} \int_{\partial \Omega_{\mu}^{0}} \mathbf{t} \otimes \mathbf{X}_{\mu} d \Gamma
$$

together with (28) we have:

$$
\begin{aligned}
& \overline{\mathbf{P}} \overline{\mathbf{F}}^{T}=\frac{1}{\Omega_{\mu}^{0}} \int_{\partial \Omega_{\mu}^{0}}\left(\mathbf{t} \otimes \mathbf{X}_{\mu}\right) \overline{\mathbf{F}}^{T} d \Gamma=\frac{1}{\Omega_{\mu}^{0}} \int_{\partial \Omega_{\mu}^{0}}\left[\overline{\mathbf{F}}\left(\mathbf{X}_{\mu} \otimes \mathbf{t}\right)\right]^{T} d \Gamma \\
& =\frac{1}{\Omega_{\mu}^{0}} \int_{\partial \Omega_{\mu}^{0}}\left[\left(\overline{\mathbf{F}} \mathbf{X}_{\mu}\right) \otimes \mathbf{t}\right]^{T} d \Gamma=\frac{1}{\Omega_{\mu}^{0}} \int_{\partial \Omega_{\mu}^{0}}\left[\mathbf{x}_{\mu} \otimes \mathbf{t}\right]^{T} d \Gamma=\frac{1}{\Omega_{\mu}^{0}} \int_{\partial \Omega_{\mu}^{0}} \mathbf{t} \otimes \mathbf{x}_{\mu} d \Gamma
\end{aligned}
$$

Using (30) we finally obtain:

$$
\frac{1}{\Omega_{\mu}^{0}} \int_{\partial \Omega_{\mu}^{0}} \mathbf{t} \otimes \mathbf{x}_{\mu} d \Gamma=\frac{1}{\Omega_{\mu}^{0}} \int_{\partial \Omega_{\mu}^{0}}\left[\mathbf{x}_{\mu} \otimes \mathbf{t}\right]^{T} d \Gamma
$$

And thus:

$$
\overline{\mathbf{P}} \overline{\mathbf{F}}^{T}=\left(\overline{\mathbf{P}} \overline{\mathbf{F}}^{T}\right)^{T}
$$




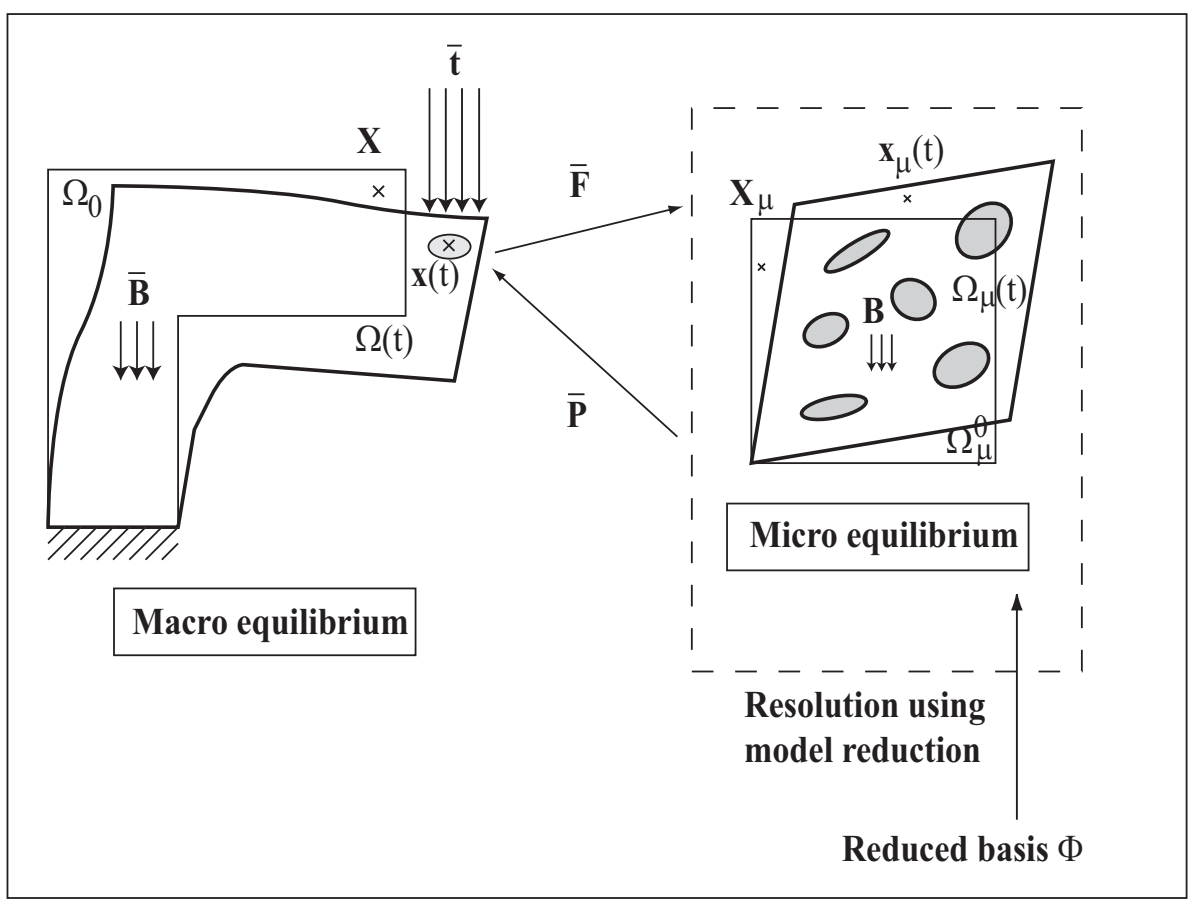

Fig. 1. R3M resolution scheme.

Table 1. Equations of the multilevel analysis.

\begin{tabular}{cc}
\hline Macro problem & Micro problem \\
$\int_{\Omega_{0}} \overline{\mathbf{P}}(\mathbf{u}): \boldsymbol{\nabla}_{X}(\boldsymbol{\delta} \mathbf{u})=\delta \bar{W}_{\text {ext }}$ & $\int_{\Omega_{0}^{\mu}} \mathbf{P}\left(\mathbf{u}_{\mu}\right): \boldsymbol{\nabla}_{X}(\boldsymbol{\delta} \mathbf{u})=\delta W_{\text {ext }}^{\mu}$ \\
\hline$\overline{\mathbf{P}}=\frac{1}{\Omega_{0}^{\mu}} \int_{\Omega_{0}^{\mu}} \mathbf{P}\left(\mathbf{u}_{\mu}\right) d \Omega$ & Boundary conditions on $\partial \Omega_{0}^{\mu}$ via $\overline{\mathbf{F}}(\mathbf{x})$ \\
\hline
\end{tabular}

which confirms the symmetry of the macroscopic Kirchhoff stress by means of the boundary conditions (28).

The coupling between the micro and macro problems is illustrated in figure 1 . The different equations are outlined in Table 1.

It is worth noting that there is no practical way of calculating the tangent matrix associated with the macro non-linear problem. One solution is to approximate this matrix using a perturbation method [8]. Computing tangent matrix in this way requires the solution of four (2D) or six (3D) finite element problems whose cost is not negligible. In this work we have used the tangent matrix associated with the homogeneous material describing the matrix of the porous structure, for the sake of simplicity. 


\section{The reduced model multiscale method (R3M)}

The R3M is a multiscale analysis, in which the problem associated with the lower scale is solved numerically by using the POD. Following similar approaches $[58,16,61,14,57,41,42,38,8,9,62,15,28]$, the computational homogenization is performed through a nested solution scheme for the coupled multiscale numerical analysis. A numerical computation of the representative volume element is carried out simultaneously in order to obtain constitutive equations at the macroscopic scale. All non-linearities come directly from the microscale. It requires simultaneous computation of the mechanical response at two different scales: the macroscopic (which is the scale of the whole structure) and the underlying microscopic RVE at each macroscopic integration point. Macroscopic phenomenological relations are unnecessary, even in non-linear case. The macro-mechanical behaviour arises directly from what happens at the microscopic scale, phenomenological constitutive equations being written only at this scale. The main contribution of R3M is to alleviate the numerous computations associated with nonlinear micro problems, by using a model reduction method. The main ingredients of R3M are given as follow:

(1) Multilevel numerical analysis;

(2) pre-computations on the RVE in order to obtain the reduced basis;

(3) resolution of the micro problem using POD;

The formulation of (1) has been presented in section 3. A detailed presentation of points (2) and (3) is made in the next sections.

\subsection{Pre-computations of the reduced basis}

In order to obtain a reduced basis which approximates reasonably the full model, it is crucial to define precisely the pre-computations that will generate the basis. Usually, several simulations are performed for different values of parameters describing the model, e.g. those associated with boundary conditions, or with material parameters. In our specific problem, the model is defined by the four parameters associated with the boundary conditions on the RVE, i.e. the four components of the macroscopic tensor $\overline{\mathbf{F}}$. One arising question is to determine the different combinations of parameters (evolving in time) that will generate an accurate solution with the obtained reduced basis. In the present context, we propose a minimal number of sampling simulations in order to construct the reduced basis. For that purpose, we propose the notion of kinematically minimal basis, i.e. the one that can reproduce exactly the essential boundary conditions.

In our specific problem, the boundary conditions on the RVE are defined 
according to (28). We thus have:

$$
\left.\mathbf{u}\right|_{\partial \Omega_{\mu}^{0}}=[\mathbf{F}-\mathbf{1}] \mathbf{X}_{\mu}=\left[\begin{array}{cc}
\gamma_{11} & \gamma_{12} \\
\gamma_{21} & \gamma_{22}
\end{array}\right]\left[\begin{array}{c}
X_{1 \mu} \\
X_{2 \mu}
\end{array}\right]
$$

We then define the following loading tests on the RVE:

$$
\begin{aligned}
& \mathbf{F}_{1}^{*}=\left[\begin{array}{cc}
\left(\gamma_{11}+1\right) & 0 \\
0 & 1
\end{array}\right] \quad \mathbf{F}_{2}^{*}=\left[\begin{array}{cc}
1 & \gamma_{12} \\
0 & 1
\end{array}\right] \\
& \mathbf{F}_{3}^{*}=\left[\begin{array}{cc}
1 & 0 \\
\gamma_{21} & 1
\end{array}\right] \quad \mathbf{F}_{4}^{*}=\left[\begin{array}{lc}
1 & 0 \\
0 & \left(1+\gamma_{22}\right)
\end{array}\right]
\end{aligned}
$$

It is worth noting that the proposed sampling simulations are necessary but not sufficient. This means that it does not guarantee that each displacement field inside of the micro domain can be reproduced, due to the nonlinear character of the problem. Additional sampling experiments may be carried out, in order to improve the accuracy of the POD solution, including other combinations of the parameters. As reported in [33], the focused data sampling leads to a very accurate reduced model, but does not lead to a reduced-order basis that can accurately capture the solution space for a range of parameter space. It has been shown in (see i.e. [33]) that in the general case the POD cannot be expected to approximate well the response away from the response paths generated individually by the evolution of the different parameters. For that purpose, an adaptation of the basis may improve the method. Several techniques have been proposed to alleviate this drawback of POD, see e.g. $[60,55,32]$. Such developments are beyond the scope of this study.

We recommend to choose the amplitude of the parameters associated with the boundary conditions such that they cover the range of applications. The procedure is described below.

We choose the amplitudes of $\gamma_{i j}$ by performing a preliminary simulation on the macrostructure. A simulation at the macro scale is carried out using the Mooney-Rivlin model (20) in order to determine $\overline{\mathbf{P}}$ at each integration points. We thus store the maximum and minimum values of each components of $\overline{\mathbf{F}}$, the amplitudes of $\gamma_{i j}$ are then chosen according to:

$$
\gamma_{i j} \in\left\{F_{i j}^{\min }-\delta_{i j} ; F_{i j}^{\max }-\delta_{i j}\right\}
$$

which gives an estimation of the amplitudes associated with the boundary condition parameters. In the above, $\gamma_{i j}$ can be either positive or negative. 
Box 1. Pre-computations of the reduced basis

1. FOR each test case $\alpha$ (see section 4.1)

1.1. Solve, for $t=t_{1}, t_{2}, \ldots, t_{S}$, the problem (19) using standard procedures

Collect the centred vectors as columns of the matrix $\mathbf{U}_{\alpha}$

$$
\mathbf{U}_{\alpha}=\left\{\mathbf{q}\left(t_{1}\right)-\overline{\mathbf{q}}, \mathbf{q}\left(t_{2}\right)-\overline{\mathbf{q}}, \ldots, \mathbf{q}\left(t_{S}\right)-\overline{\mathbf{q}}\right\}
$$

with $\overline{\mathbf{q}}=\frac{1}{S} \sum_{i=1}^{S} \mathbf{q}\left(t_{i}\right)$

Collect the matrix $\mathbf{U}_{\alpha}$ in $\mathbf{V}$ such as:

$$
\mathbf{V}=\left\{\mathbf{U}_{1} ; \mathbf{U}_{2} ; \ldots ; \mathbf{U}_{\alpha}\right\}
$$

\section{END}

2. Construct the covariance matrix

$\mathbf{Q}=\mathbf{V} \mathbf{V}^{T}$

3. Solve the eigenvalue problem:

$\mathbf{Q} \phi_{k}=\lambda_{k} \phi_{k}$

4. Construct the reduced basis

$\Phi=\left\{\phi_{1}, \phi_{2}, \ldots, \phi_{M}\right\}$

where $M$ is chosen according to (9)

\subsection{Resolution of the micro problems using POD}

The discretization of the linearized problem associated with (19) using a Galerkin procedure, i.e. the finite element method (other methods could alternatively be considered, i.e. meshfree methods or boundary elements method) leads to the following discrete system of equations, by applying an incremental procedure:

$$
\mathbf{K}_{\mu}^{k} \Delta \mathbf{q}^{k+1}=\mathbf{f}_{e x t(\mu)}-\mathbf{f}_{i n t}^{k}\left(\mathbf{q}_{\mu}^{k}\right)
$$

where $\mathbf{K}_{\mu}^{k}$ is the tangent matrix computed from (53), $\mathbf{f}_{\text {ext }(\mu)}$ and $\mathbf{f}_{\text {int }}^{k}$ denote the internal and external forces vectors, respectively, computed from (59). In the following, The superscript $k$ denotes the iteration index, while the subscript $n$ denotes the increment index.

The central contribution of R3M is to reduce the problem (36) into a small system of linear equations using POD. Let $\Phi$ a set of basis function such as $\boldsymbol{\Phi}=\left\{\phi_{1}, \phi_{2}, \ldots, \phi_{M}\right\}$, as defined in the former section. The increment of 
displacement is expanded, by the Ritz basis of M functions $\phi_{m}$, as:

$$
\Delta \mathbf{q}^{k+1}=\sum_{m=1}^{M} \phi_{m} \Delta \xi_{m}^{k+1}
$$

Introduction of (37) in (36) leads to:

$$
\mathbf{K}_{\mu}^{k} \boldsymbol{\Phi} \Delta \boldsymbol{\xi}^{k+1}=\mathbf{f}_{e x t(\mu)}-\mathbf{f}_{i n t}^{k}\left(\mathbf{q}_{\mu}^{k}\right)
$$

where $\boldsymbol{\xi}=\left\{\xi_{1}, \xi_{2}, \ldots, \xi_{M}\right\}$. Pre-multiplication of (38) with $\boldsymbol{\Phi}^{T}$ leads to:

$$
\boldsymbol{\Phi}^{T} \mathbf{K}_{\mu}^{k} \boldsymbol{\Phi} \Delta \boldsymbol{\xi}^{k+1}=\boldsymbol{\Phi}^{T}\left[\mathbf{f}_{e x t(\mu)}-\mathbf{f}_{i n t}^{k}\left(\mathbf{q}_{\mu}\right)\right]
$$

After resolution of (39), the reduced variables are updated according to:

$$
\xi^{k+1}=\xi^{k}+\Delta \xi^{k+1}
$$

We note that : (a) the resolution of (39) only involves the inversion of a $M \times M$ matrix instead of a $N D \times N D$ for the full problem, with $M<<N D$ (we recall that $N$ and $D$ denote the number of nodes and the space dimension, respectively); (b) the storage of $\boldsymbol{\xi}^{k}$ at each integration point involves only $M \times P$ real number table, with $P$ the number of integration points on the macro structure, $P$ being of the order of $N$. If three scales are involved, the size of the database in the full multilevel method (i.e. $\mathrm{FE}^{2}[8,9]$ growths with $O\left(N^{3}\right)$, with $N$ the number of nodes, assumed to be of the same order in the micro and micro problems. In the R3M, the complexity of the database remains $O\left(M^{2} N\right)$. It is worth noting that such simulation would still involve large computations in both approaches. Nevertheless, the R3M is fully compatible with parallel approaches, but remains less expensive than full approaches, as it will be demonstrated in the next section.

\subsection{General algorithm of R3M}

The general algorithm is outlined in Box 1, 2, and 3. A schematic view of the resolution strategy is depicted in figure 1. In Box 2 and $3, \mathbf{q}$ denotes the generalized displacement vector, while $\mathbf{u}$ denotes the displacement vector associated with one element containing an integration point. 
LOOP over all time steps

Given $\mathbf{q}_{n}$ :

Initialize $\mathbf{q}_{n+1}=\mathbf{q}_{n}$ :

WHILE $\|\mathbf{R}\|>T O L \quad\left(\mathbf{R}=\mathbf{f}_{e x t}-\mathbf{f}_{i n t}\right)$

LOOP over all integration points $\mathbf{X}_{i}$ :

Compute $\overline{\mathbf{F}}\left(\mathbf{X}_{i}\right)=\mathbf{1}+\boldsymbol{\nabla}_{X}\left(\mathbf{u}_{n+1}^{k}\right)$

Given $\left[\boldsymbol{\xi}\left(\mathbf{X}_{i}\right)\right]_{n}$, compute $\overline{\mathbf{P}}\left(\mathbf{X}_{i}\right)$ and $\left[\boldsymbol{\xi}\left(\mathbf{X}_{i}\right)\right]_{n+1}$ from the micro domain; go to Box 3 .

Compute the elementary contributions $\left[\mathbf{f}_{i n t(e)}\left(\mathbf{X}_{i}\right)\right]_{n+1}^{k+1},\left[\mathbf{K}_{T(e)}\left(\mathbf{X}_{i}\right)\right]_{n+1}^{k+1}$ and assemble them in $\left[\mathbf{f}_{i n t}\right]_{n+1}^{k+1}$ and $\left[\mathbf{K}_{T}\right]_{n+1}^{k+1}$

\section{END}

Solve $\left[\mathbf{K}_{T}\right]_{n+1}^{k+1} \Delta \mathbf{q}_{n+1}^{k+1}=\mathbf{f}_{e x t}-\left[\mathbf{f}_{i n t}\right]_{n+1}^{k+1}$

Update displacements:

$\mathbf{q}_{n+1}^{k+1}=\Delta \mathbf{q}_{n+1}^{k+1}+\mathbf{q}_{n+1}^{k}, \quad k \leftarrow k+1$

\section{END}

END

\section{$5 \quad$ Numerical examples}

\subsection{Analysis on some various representative volume elements}

The main objective of the proposed approach is to solve the microscopic problem using a POD model reduction method. As described in section 4.1, the reduced basis is generated by performing some simulations and then collecting snapshots of the solution so as to select influent modes (see Box 1). Nevertheless, these pre-computations are carried out for some specific values of parameters describing the model. In the present context, these parameters are the four components of the 2D-macroscopic deformation tensor $\overline{\mathbf{F}}$, which define the boundary conditions on the RVE through the relation (28). In the multiscale analysis (see Boxes 2 and 3), the microscopic problem is solved using arbitrary combinations of the boundary conditions, provided by the macroscopic scale, that do not necessarily match the values of the parameters used in the pre-computations. The aim of this first example is two fold: on one hand, we evaluate the accuracy of the solution provided by the reduced model, away from the parameter paths used in the pre-computations; on the other hand, we 
Box 3. Resolution of the MICRO problem

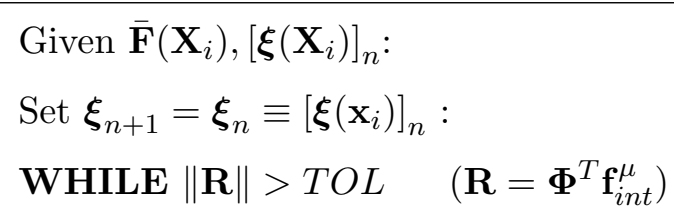

Reconstruct displacement field in the micro domain from the reduced basis:

$\left[\mathbf{q}_{\mu}\right]_{n+1}^{r}=\boldsymbol{\Phi} \boldsymbol{\xi}_{n+1}^{r}$

LOOP over all integration points $\left[\mathbf{X}_{\mu}\right]_{j} \equiv \mathbf{X}_{j}^{\mu}$ :

Compute $\mathbf{F}\left(\mathbf{X}_{j}^{\mu}\right)=\mathbf{1}+\boldsymbol{\nabla}_{X}\left(\left[\mathbf{u}_{\mu}\right]_{n+1}^{r}\right)$

Compute $\mathbf{P}\left(\mathbf{X}_{j}^{\mu}\right)$ from constitutive equation

Compute the elementary contributions $\left[\mathbf{f}_{i n t(e)}^{\mu}\left(\mathbf{X}_{j}^{\mu}\right)\right]_{n+1}^{r+1},\left[\mathbf{K}_{T(e)}^{\mu}\left(\mathbf{X}_{j}^{\mu}\right)\right]_{n+1}^{r+1}$ and assemble them in $\left[\mathbf{f}_{i n t}^{\mu}\right]_{n+1}^{r+1},\left[\mathbf{K}_{T}^{\mu}\right]_{n+1}^{r+1}$

END

Solve the reduced problem and update reduced variables:

$\boldsymbol{\Phi}^{T}\left\{\left[\mathbf{K}_{T}^{\mu}\right]_{n+1}^{r+1}\right\} \boldsymbol{\Phi} \boldsymbol{\Delta} \boldsymbol{\xi}_{n+1}^{r+1}=-\boldsymbol{\Phi}^{T}\left[\mathbf{f}_{i n t}^{\mu}\right]_{n+1}^{r+1}$

$\boldsymbol{\xi}_{n+1}^{r+1}=\boldsymbol{\Delta} \boldsymbol{\xi}_{n+1}^{r+1}+\boldsymbol{\xi}_{n+1}^{r}, \quad r \leftarrow r+1$

\section{END}

$\left[\boldsymbol{\xi}\left(\mathbf{x}_{i}\right)\right]_{n+1}=\boldsymbol{\xi}_{n+1}^{r+1}$

Compute the average stress in the micro domain:

$\overline{\mathbf{P}}\left(\mathbf{X}_{i}\right)=\frac{1}{\Omega_{\mu}^{0}} \int_{\Omega_{\mu}^{0}} \mathbf{P}\left(\mathbf{X}_{\mu}\right) d \Omega$

aim at evaluating the influence of some features of the model on the accuracy of the POD solution and on the number of selected modes. These features are: (a) the geometrical complexity of the domain; (b) the constitutive model; (c) the number of pre-computations used to construct the reduced basis.

To test the influence of the geometrical complexity, four models of RVE have been constructed, as depicted in figures 2 (a)-(d), with increasing complexity. In the following, these models are referred to as RVE1, RVE2, RVE3 and RVE4, respectively.

Two material models are used: (a) a Mooney-Rivlin model described by Eq. (20) with parameters $c_{1}=6.3 .10^{5} \mathrm{~N} / \mathrm{m}^{2}, c_{2}=-0.012 .10^{5} \mathrm{~N} / \mathrm{m}^{2}$ and $c=$ $20.10^{5} \mathrm{~N} / \mathrm{m}^{2}$ and (b) A Neo-Hookean model described by the Eq. (20) with parameters $c_{1}=6.3 .10^{5} \mathrm{~N} / \mathrm{m}^{2}, c_{2}=0 \mathrm{~N} / \mathrm{m}^{2}$ and $c=100.10^{5} \mathrm{~N} / \mathrm{m}^{2}$. The second model is nearly incompressible, and thus involves more geometrical non-linearities. These two models will be used to determine the influence of the geometrical nonlinearity on the number of selected basis. 


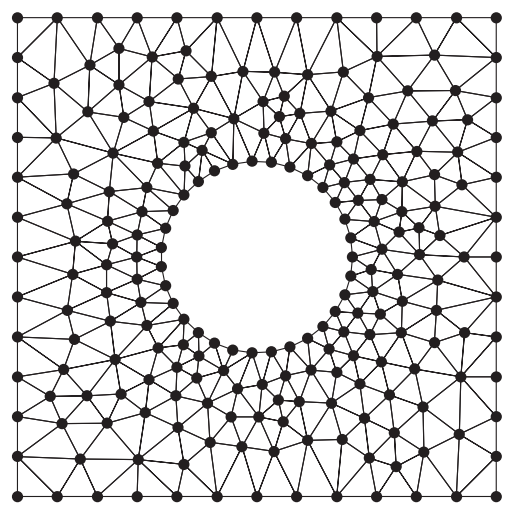

(a)

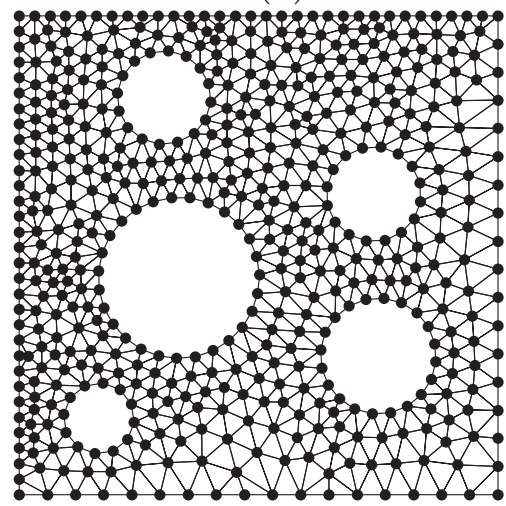

(c)

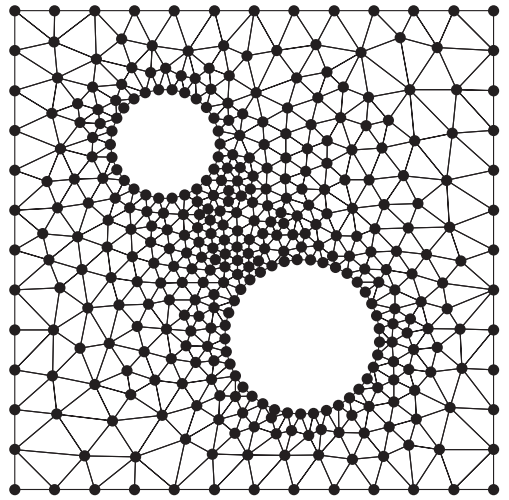

(b)

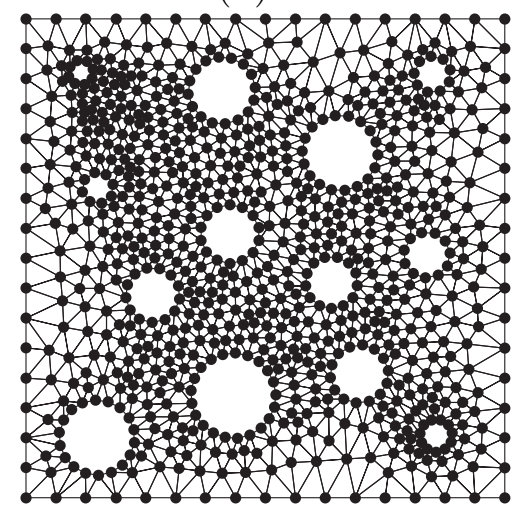

(d)

Fig. 2. Different RVE used for the analysis (a) RVE1, 452 d.o.f.; (b) RVE2, 744 d.o.f; (c) RVE3, 1056 d.o.f.; (d) RVE4, 1612 d.o.f;

Two sampling experiments are carried out to construct the reduced basis. In the first experiment, four simulations are conducted, in which each of the four parameters describing the boundary conditions varies independently. The reduced basis is then constructed using the set of obtained samples, as described in Box 1. In the second experiment, an additional simulation is performed, combining several parameters, whose evolution is described in figure 3 . In the second case, the reduced basis is constructed using five samplings. The resutling numbers of modes for $\delta=1.10^{-7}$ in Eq. (9) and $\gamma=0.25$ are given in Table 2, $\gamma$ being described in figure 3. The first nine representative modes associated with sampling experiment 2, RVE4 and Neo-Hookean model, are depicted in figure 4 . The associated normalized eigenvalues $\beta_{i}=\lambda_{i} / \lambda_{\max }$ are indicated.

From Table 2, we can notice that for the proposed example, the number of modes is almost independent of the number of degrees of freedom of the full model, but is also independent of the geometrical complexity of the full model. Nevertheless, we can observe that the size of the reduced basis is larger for the Neo-Hookean model. This can be explained by the fact that an increase in the material parameter $c$ in Eq. (20) induces a higher level of incompressibility, which leads to more geometrical non-linearities. For both material models, 


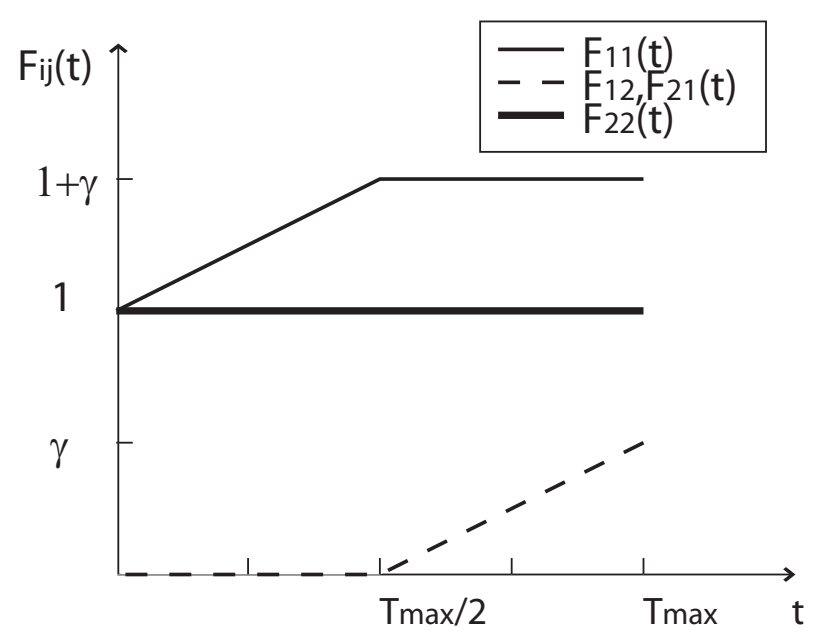

Fig. 3. Evolution of the components of the boundary conditions used in the precomputations.

Table 2. Analysis of the reduced basis size.

\begin{tabular}{lllllll}
\hline & & \multicolumn{2}{c}{$\begin{array}{l}\text { 4 sampling } \\
\text { simulations }\end{array}$} & \multicolumn{3}{c}{$\begin{array}{l}\text { s sampling } \\
\text { simulations }\end{array}$} \\
\hline \multirow{2}{*}{ Model } & $\begin{array}{l}\text { Total nb. } \\
\text { of d.o.f. }\end{array}$ & $\begin{array}{l}\text { Nb. reduced } \\
\text { modes }\end{array}$ & $\begin{array}{l}\text { Max. } \\
\text { error } \%\end{array}$ & $\begin{array}{l}\text { nb. reduced } \\
\text { modes }\end{array}$ & $\begin{array}{l}\text { Max. } \\
\text { error } \%\end{array}$ \\
\hline RVE 1 & Moon.-Riv. & 452 & 18 & $1.5 \%$ & 22 & $1.4 \%$ \\
& Neo-Hook. & & 21 & $7 \%$ & 25 & $2.5 \%$ \\
\hline \multirow{2}{*}{ RVE 2} & Moon.-Riv. & 744 & 19 & $3 \%$ & 23 & $2.2 \%$ \\
& Neo-Hook. & & 22 & $8 \%$ & 25 & $3 \%$ \\
\hline \multirow{2}{*}{ RVE 3} & Moon.-Riv. & \multirow{2}{*}{1056} & 19 & $1.8 \%$ & 23 & $1.5 \%$ \\
& Neo-Hook. & & 23 & $15 \%$ & 26 & $4.6 \%$ \\
\hline \multirow{2}{*}{ RVE 4} & Moon.-Riv. & \multirow{2}{*}{1612} & 21 & $3.1 \%$ & 24 & $1.8 \%$ \\
& Neo-Hook. & & 25 & $12 \%$ & 29 & $4.7 \%$ \\
\hline
\end{tabular}

the number of modes is higher when adding the sampling experiment with the parameter evolution described in figure 3. It is shown that the resulting displacement fields can not be reproduced accurately with the minimal basis, obtained with independent evolution of the parameters.

In order to test the accuracy of the reduced basis away from the sampling paths, we impose boundary conditions on the different RVE using the evolution of the different parameters $\bar{F}_{11}, \bar{F}_{12}, \bar{F}_{21}$ and $\bar{F}_{22}$ as depicted in figure 5 . The different curves describing the evolution of the parameters with respect to time have been chosen as quadratic functions, such as the load passes continuously 


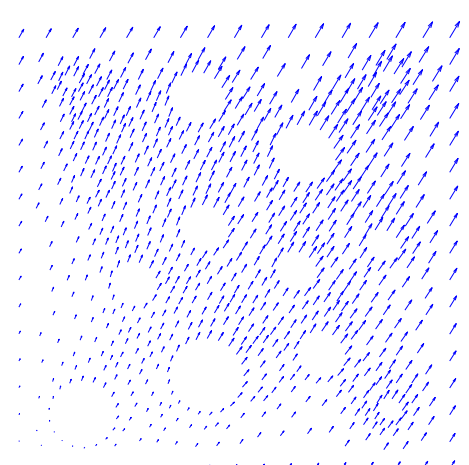

$$
\beta_{1}=1.00
$$

$$
\beta_{2}=2.12 .10^{-1}
$$

$\beta_{3}=4.35 .10^{-2}$
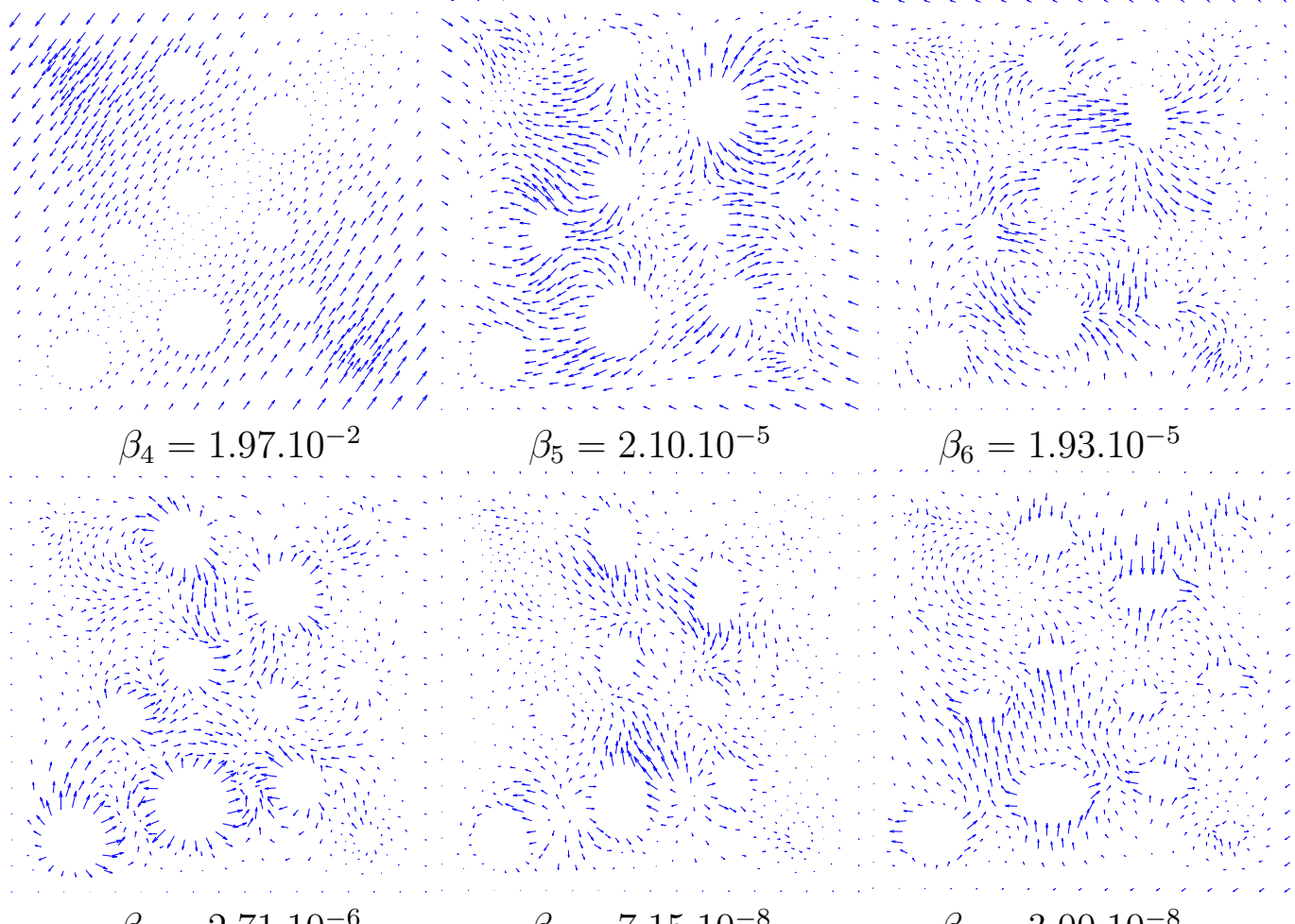

$$
\beta_{7}=2.71 .10^{-6}
$$

$$
\beta_{8}=7 \cdot 15 \cdot 10^{-8}
$$

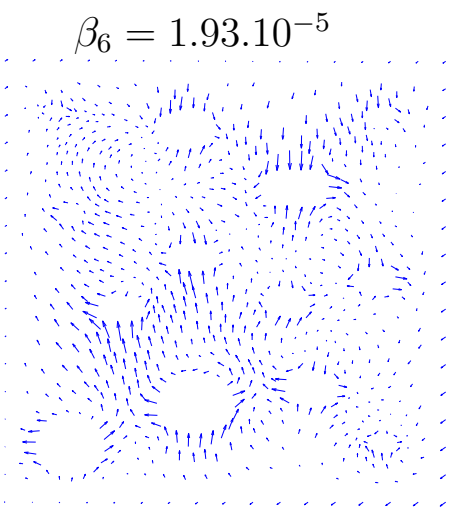

Fig. 4. First nine modes of the reduced basis using RVE4.

from a pure biaxial traction state, for $t=T_{\max } / 2$, to a complex combined shear/traction loading, for $t=T_{\max }$.

The deformed configurations of the RVE 4 with Neo-Hookean model corresponding to full analysis (1612 dof) and reduced model (29 dof) are compared in figure 6 . We can observe that the values of stress are similar. For a more in-depth analysis of the accuracy, the relative error:

$$
e=100 \frac{P_{i j}^{\text {full }}-P_{i j}^{\text {reduced }}}{\left|P_{i j}^{f u l l}\right|}
$$

between full and reduced analysis has been computed for each test of Table 2 . 

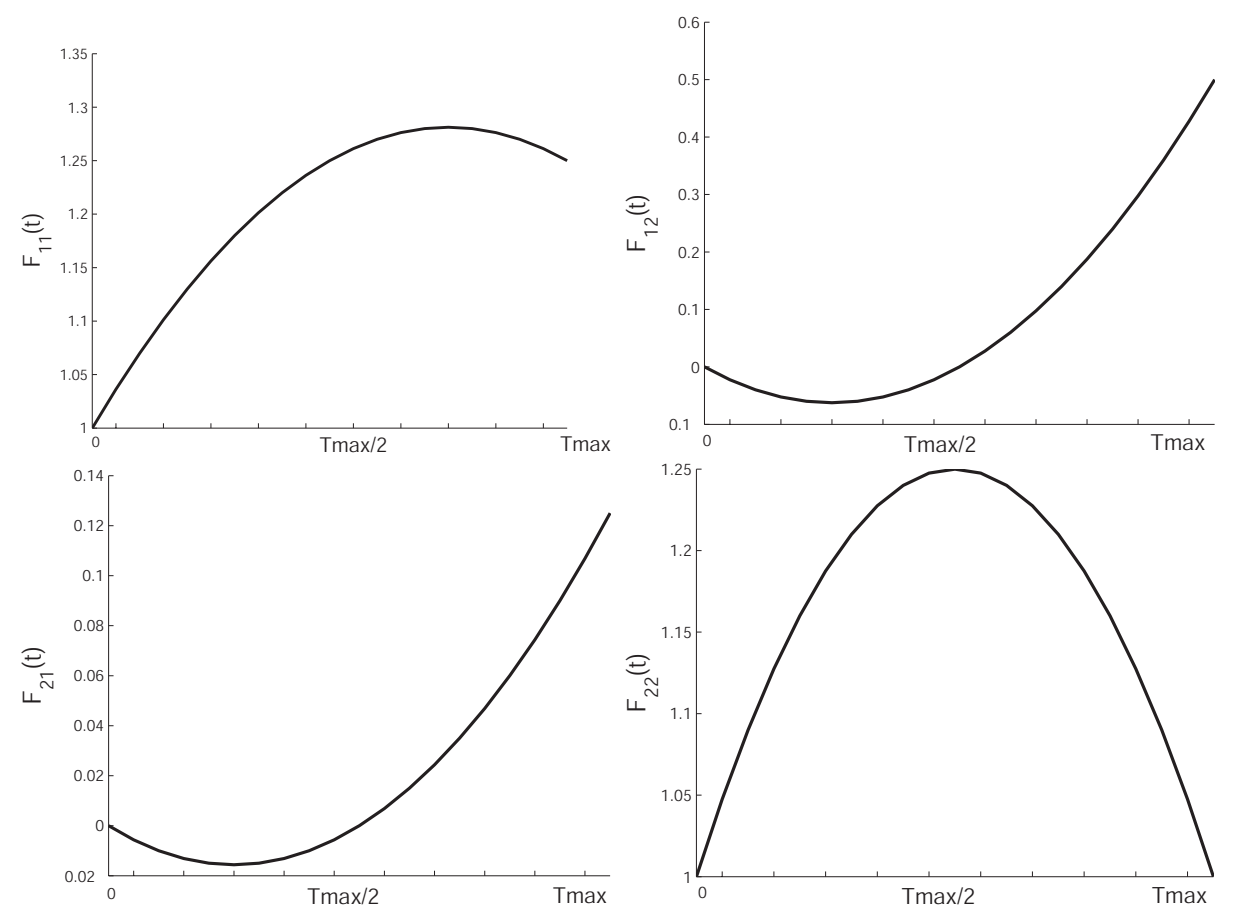

Fig. 5. Values of $F_{11}, F_{12}, F_{21}$ and $F_{22}$ along the simulation.
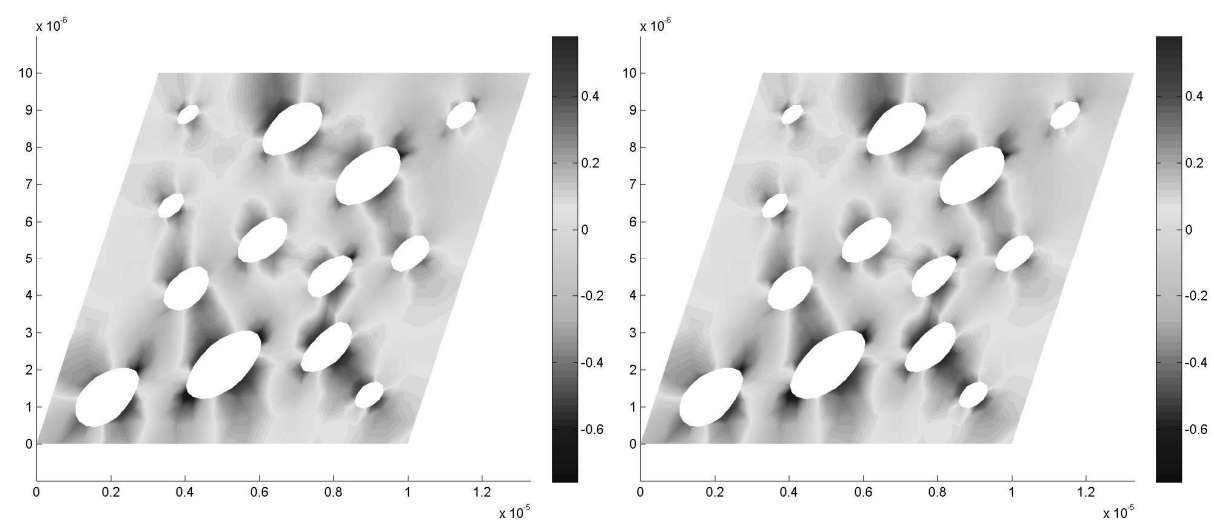

Fig. 6. Deformed configurations of RVE 4 at time $t=T_{\max }$ : (a) full analysis (1612 d.o.f.); (b) reduced model (29. d.o.f.)

The results are indicated in Table 2. We can observe that the reduced model constructed with the first sampling experiment induces higher errors than with the second sampling experiment, which implies an additional sampling path. A comparison of the homogenized stresses obtained for a particular case, by choosing RVE 3 and Neo-Hookean model, is provided in figure 7.

From figure 7 (a), we can notice that for $t>T_{\max } / 2$, there is a divergence between the reduced and full model solutions, which suggests that the basis generated by the evolution of the parameters can not reproduce accurately the solution for the Neo-Hookean model. The reduced basis produced by the additional sampling experiment provides a much better solution, as can be seen 


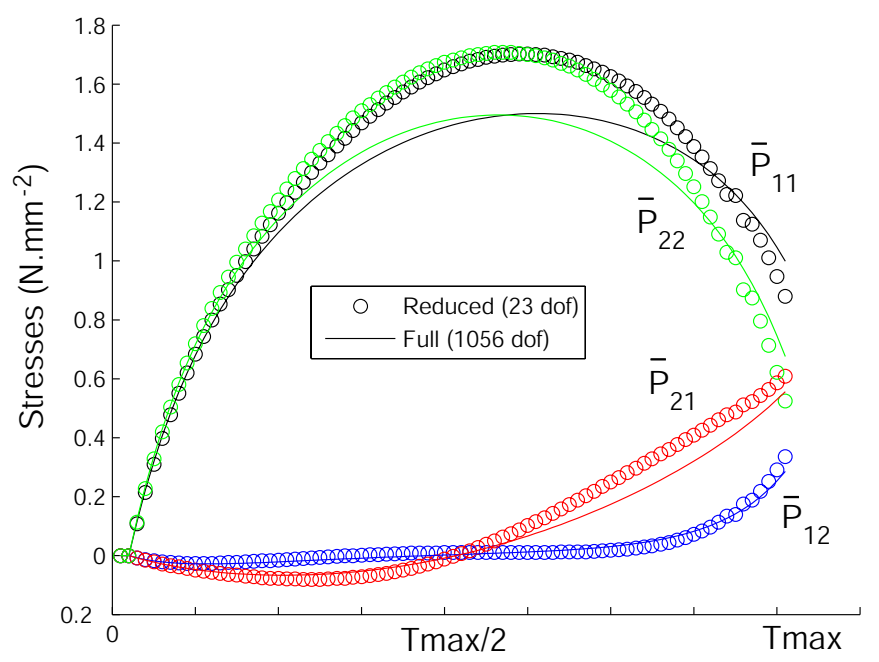

(a)

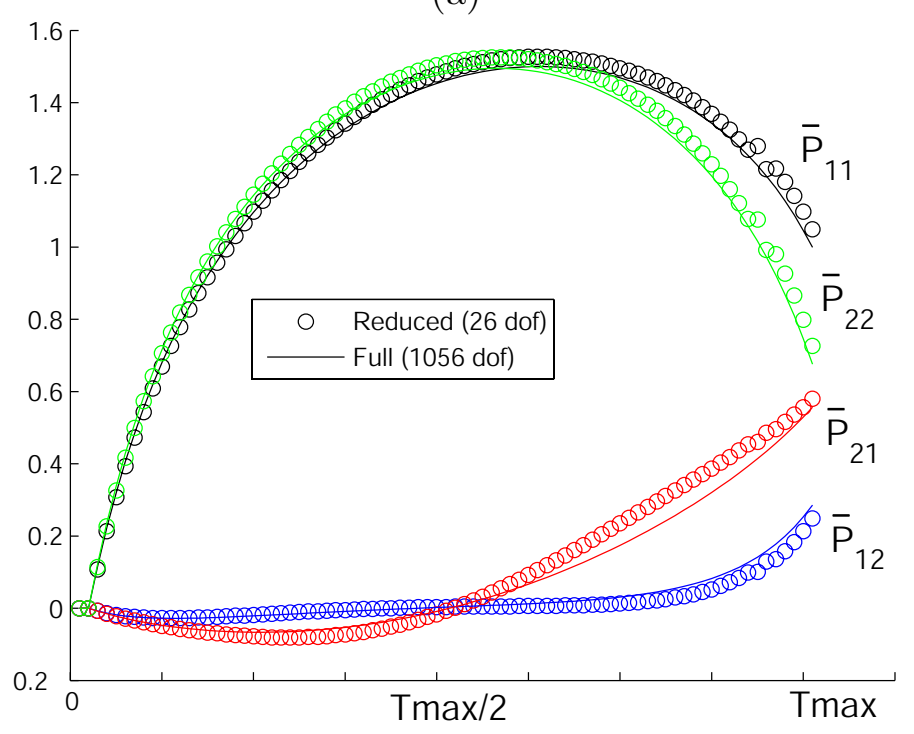

(b)

Fig. 7. Comparison between full and reduced solutions.

in figure 7 (b). A complete study for the different geometrical and material models is provided in Table 2. The same conclusions can be drawn for the different RVE models. The error is much smaller for the Mooney-Rivlin model, because the geometrical non-linearities are lower for this model. Thus, the biaxial traction state does not give rise to much additional uncorrelated modes. 


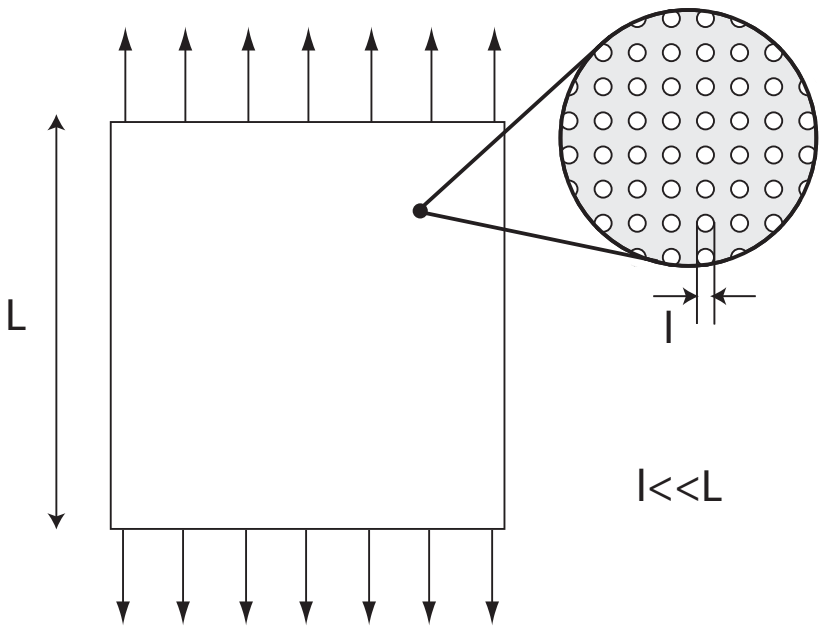

(a)

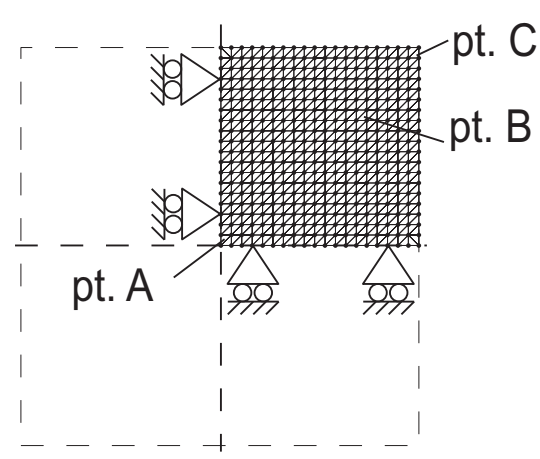

(b)

Fig. 8. (a) Geometry of the problem; (b) finite element model.

\subsection{Traction test}

In the former example, no coupling between the micro and macro scales has been considered. Here a complete multi-scale analysis is carried out. A porous material whose matrix is characterized by the compressible Mooney-Rivlin model (20) considered. The following numerical parameters are chosen: $c_{1}=$ $6.3 .10^{5} \mathrm{~N} / \mathrm{m}^{2}, c_{2}=-0.012 .10^{5} \mathrm{~N} / \mathrm{m}^{2}$ and $c=20.10^{5} \mathrm{~N} / \mathrm{m}^{2}$.

The diameters of the holes are taken to be very small compared to the characteristic length of the specimen. The macro structure consists of a twodimensional square plate submitted to plane strains traction, as depicted in figure 8 (a). Due to the symmetry of the problem, only a quarter of the plate is modelled. The FE model of the macrostructure is depicted in figure 8 (b), and consists in 400 nodes and a total of 722 integration points.

In order to perform a multiscale analysis, we associate a representative volume element (RVE) with each integration point of the macrostructure FE model. The FE model of the RVE is shown in figure 2. The porosity of the material is 0.03 . The full FE model of the RVE involves 832 degrees of freedom. Displacements on the boundary $\left(X_{2}=L / 2\right)$ of the macrostructure are imposed, corresponding to stretches ranging from $0 \%$ to $40 \%$.

In order to obtain the reduced basis, preliminary computations are performed on the RVE, obtained by the POD procedure described in section 2 and Box 1. Here, the reduced basis has been constructed using only the minimal number of sampling experiments proposed in section 4.1. The number of basis is determined by choosing $\delta=10^{-7}$ in (9). For this particular problem, 20 modes are selected, which remains much smaller than the 832 d.o.f. of the full model. 


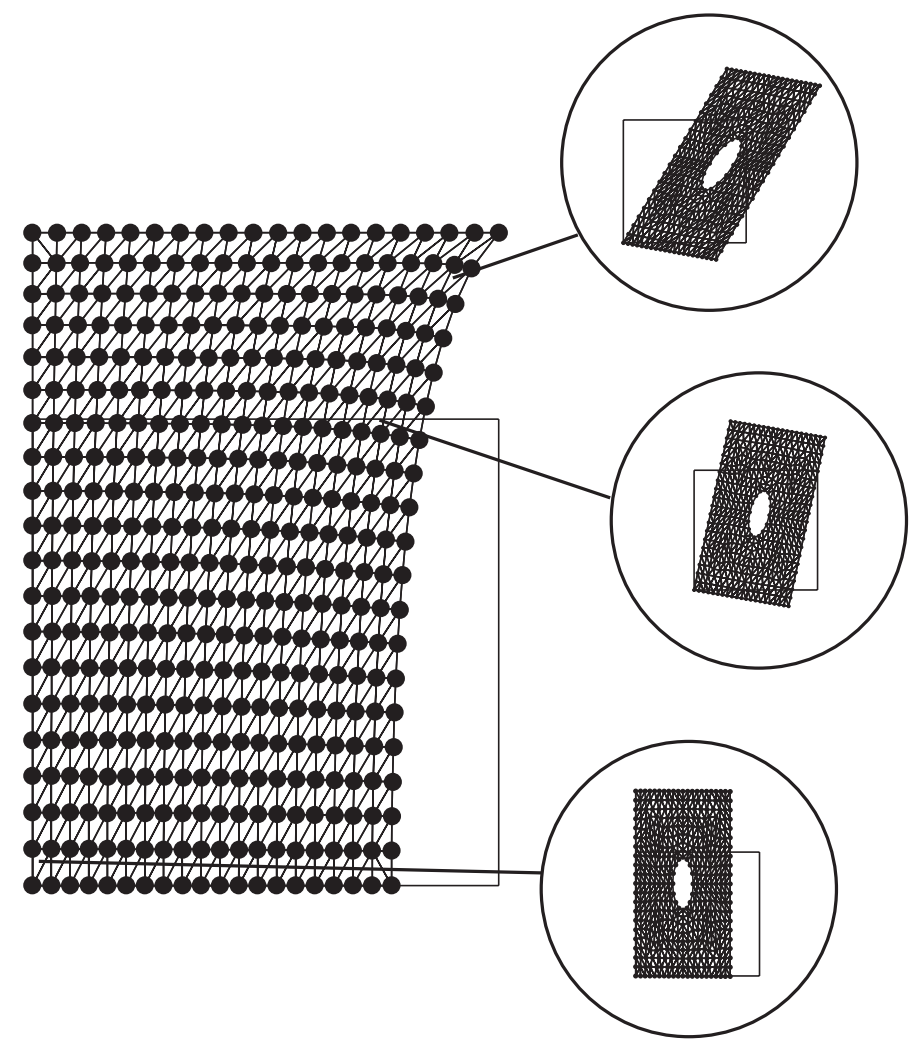

Fig. 9. Deformation of the macro and micro structures.

A full multiscale computation is performed, according to the procedure described section 4.3. We carry out two simulations, one using the full RVE model, which will be used as a reference solution, and one using the reduced model, implying only 20 modes. The deformation of the macrostructure and of the microstructures associated with points A, B and C (see figure 8 (b)) are plotted in figure 9 .

We compare for the points $\mathrm{A}, \mathrm{B}$ and $\mathrm{C}$ the homogenized stresses $\bar{P}_{11}, \bar{P}_{12}$, $\bar{P}_{21}$ and $\bar{P}_{22}$ obtained through the full and reduced approaches, during the simulation. The stresses are plotted versus the deformation of the macroscopic structure defined by: $\epsilon=2 \overline{\mathbf{u}}_{y}\left(\mathbf{x}_{2}=H\right) / L$. We recall that the same reduced basis is used for the resolution of problems associated with all the integration points of the macrostructure. The results are depicted in figures 10 (a), 11 (a) and 12 (a). The relative error in percent is given in figures 10 (b), 11 (b) and $12(\mathrm{~b})$.

Remarkably, the same reduced basis is able to reproduce accurately the kinematics associated with the arbitrary linear boundary conditions, as defined in (27 (i)). Less than $0.25 \%$ error was observed for the points studied, which are representative of the different loads in the structure.

To evaluate the accuracy of the solution with respect to the number of basis 


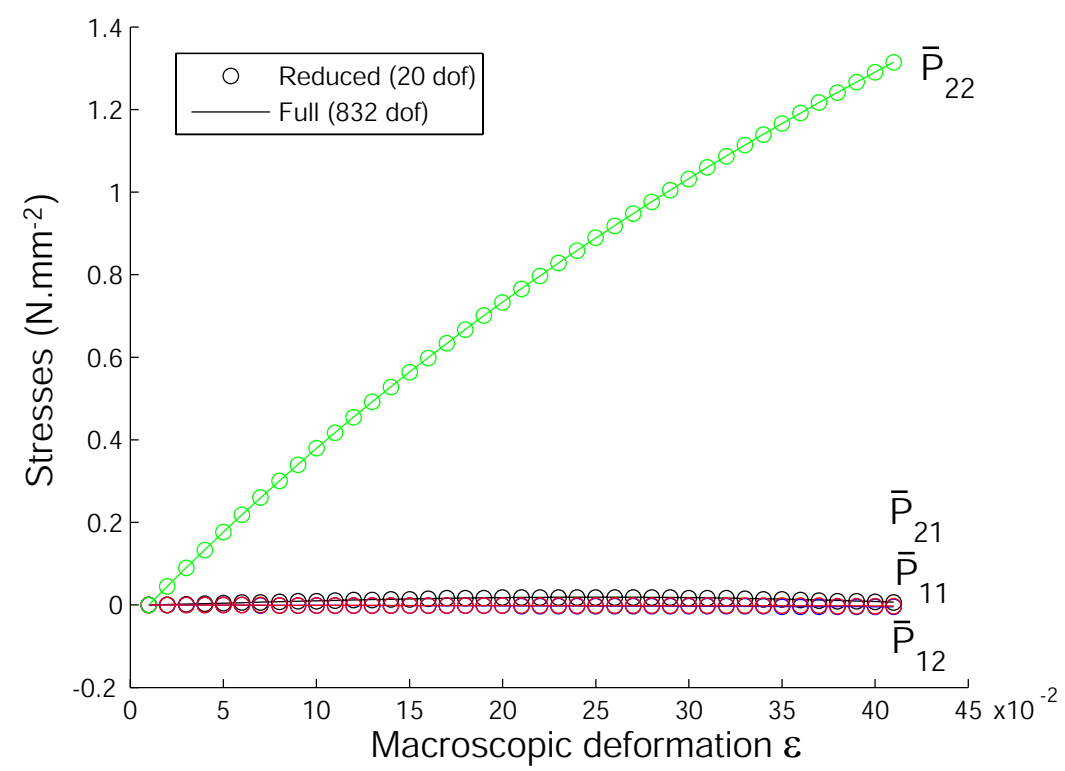

(a)

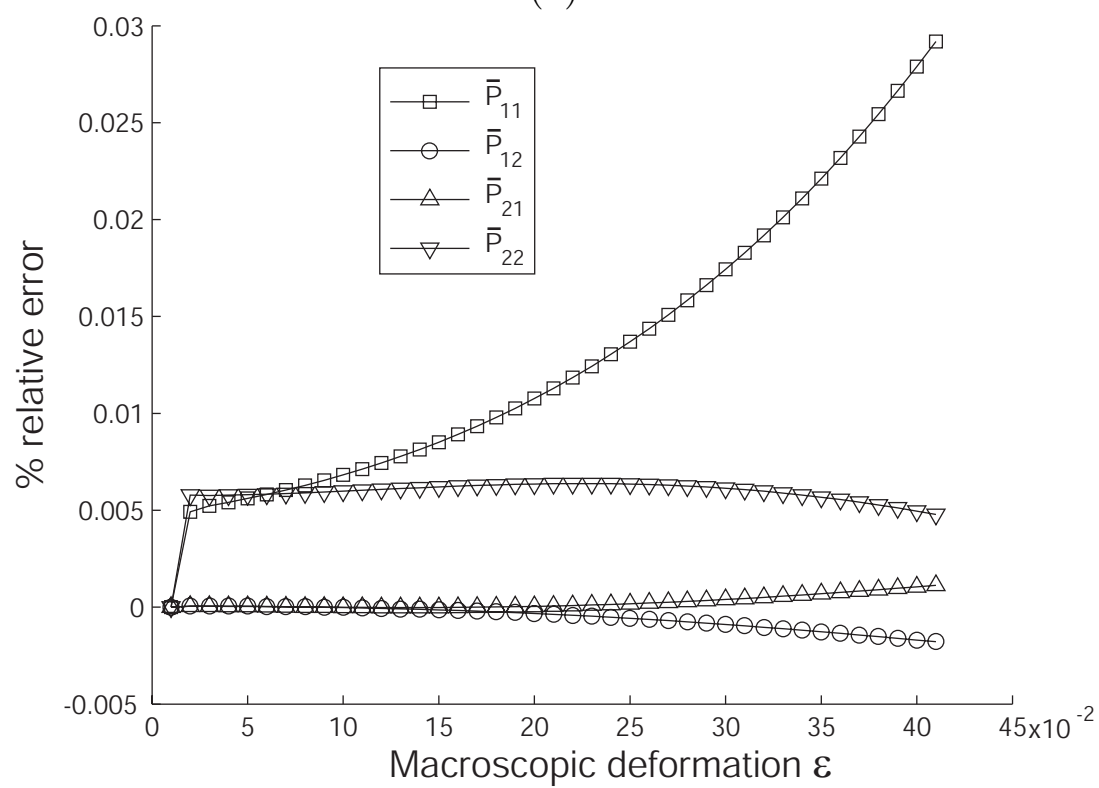

(b)

Fig. 10. Comparison between R3M and full multiscale computation (point A).

functions, a convergence analysis is carried out. We have computed, for point $\mathrm{C}$, the cumulated error:

$$
e(M)=\int_{0}^{T}\left\|P_{i j}^{r e d}(M)-P_{i j}^{f u l l}\right\| d t
$$

with respect to the number of basis functions $M$. The values of $\delta$ (see Eq. (9)) are also indicated. The results are depicted in figure 13 . 


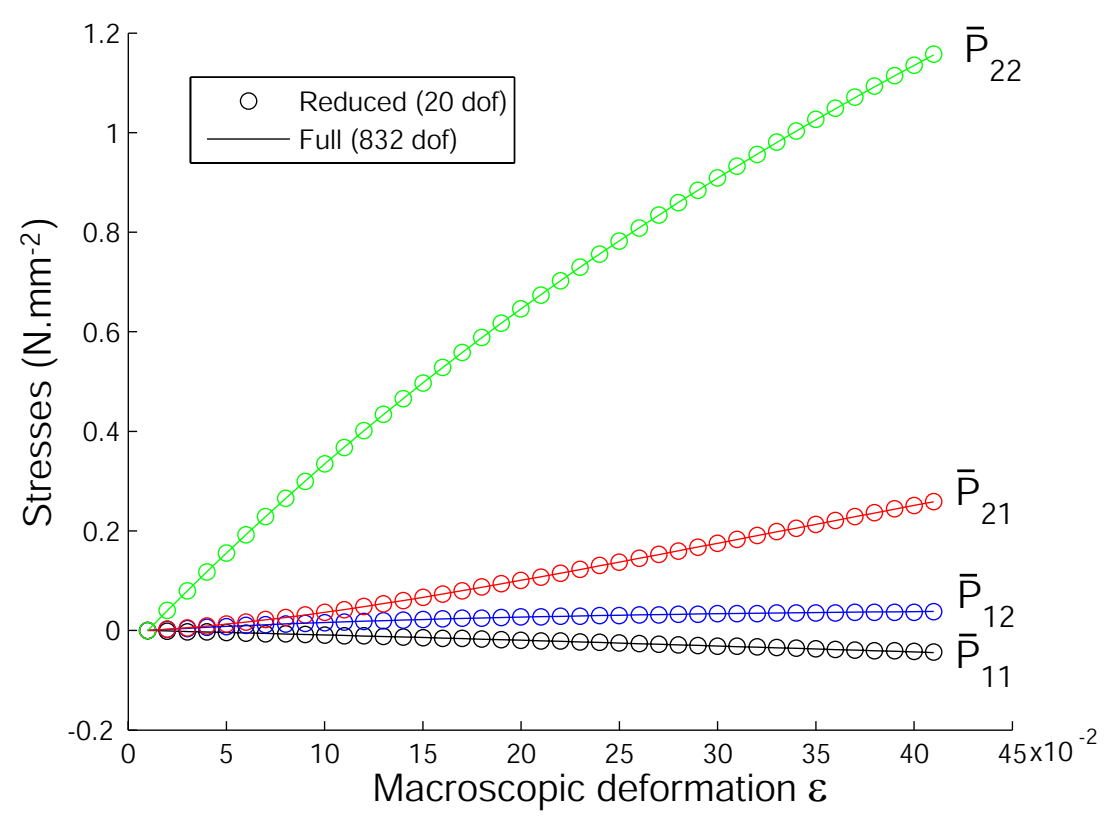

(a)

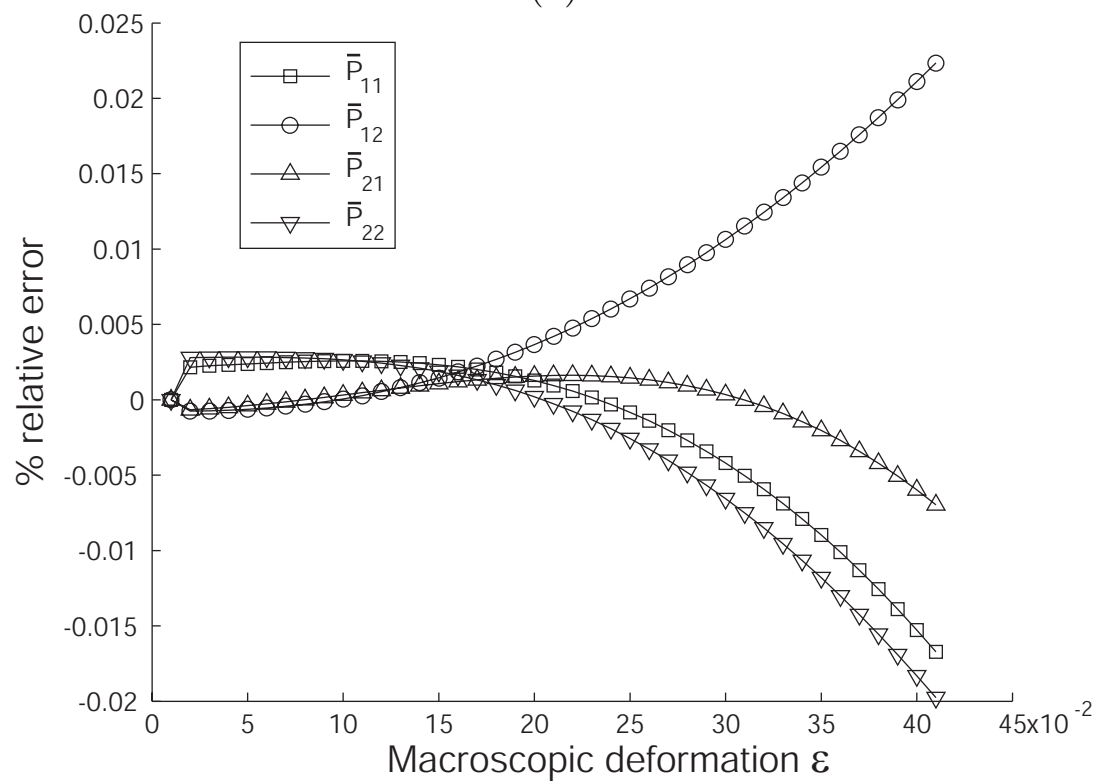

(b)

Fig. 11. Comparison between R3M and full multiscale computation (point B).

From figure 13 we can observe that the error decreases quickly with the number of basis functions. Above 20 modes $\left(\delta=10^{-7}\right)$, the gain in accuracy is not significant. Nevertheless, the value of $\delta$ is obviously problem-dependent. The question of deciding which modes should be preserved in the model has been evocated in Lall et al. [30] Typically, the low-frequency modes are kept. However there are many situations where this is not the best choice: in particular, in control systems where the frequency at which an accurate model is necessary is at crossover, and may not correspond to the low frequency modes of the system. It has also been reported in Joyner et al. [25] that in some 


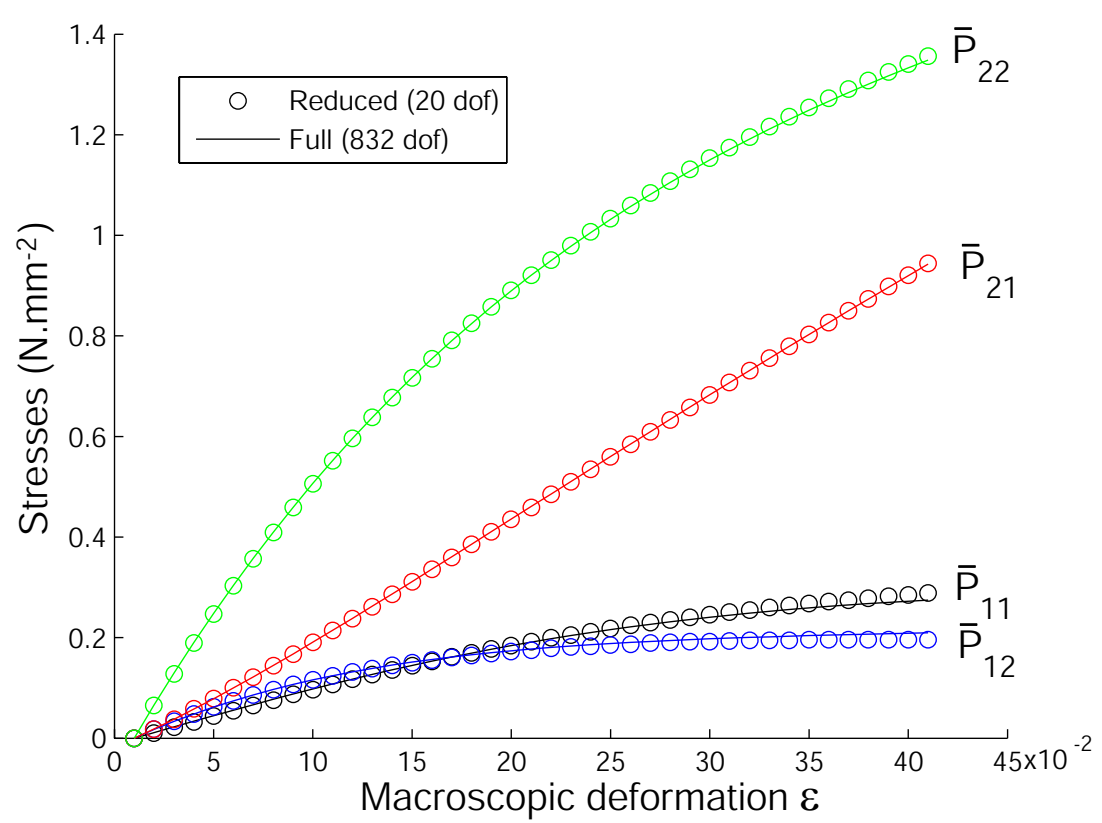

(a)

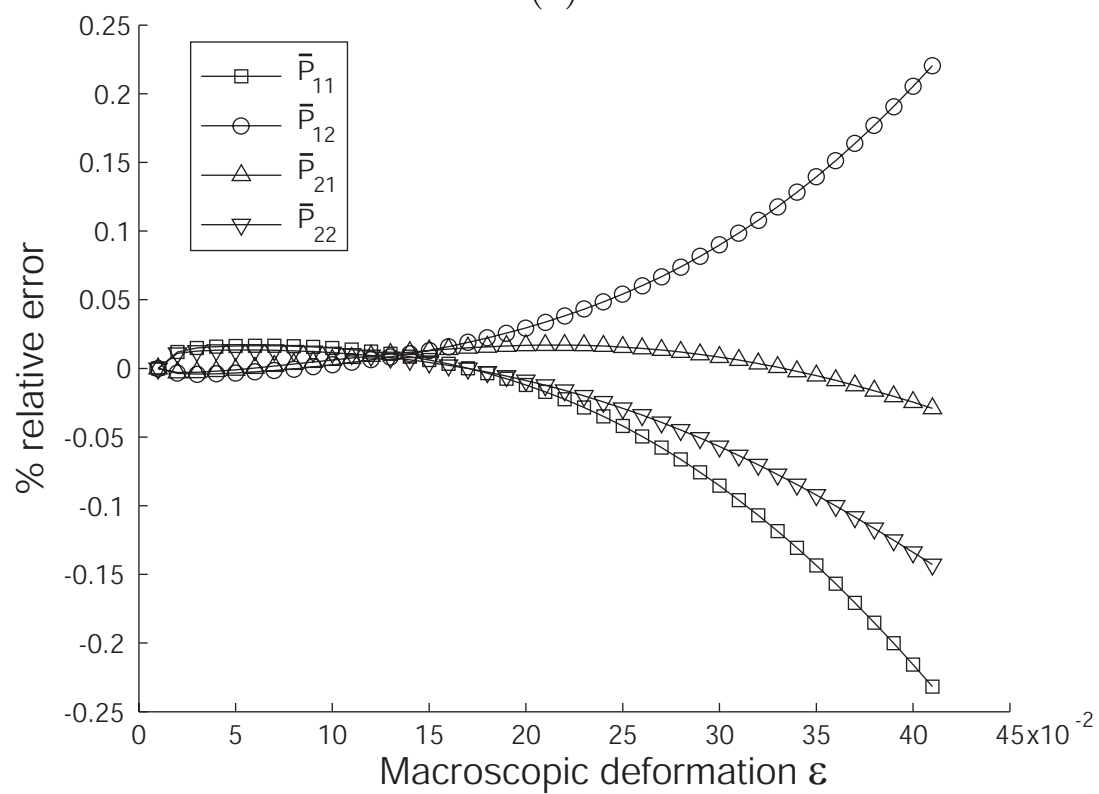

(b)

Fig. 12. Comparison between R3M and full multiscale computation (point C).

cases, that further increase of number of eigenfunctions up to the optimal number of empirical eigenfunctions does not improve accuracy and may even deteriorates accuracy because the eigenfunctions with small eigenvalues are contemned with round-off errors.

The time computations as well as the size of the database needed to describe the history of the macroproblems are given in Table 3 . The times are normalized with respect to the minimum value. 


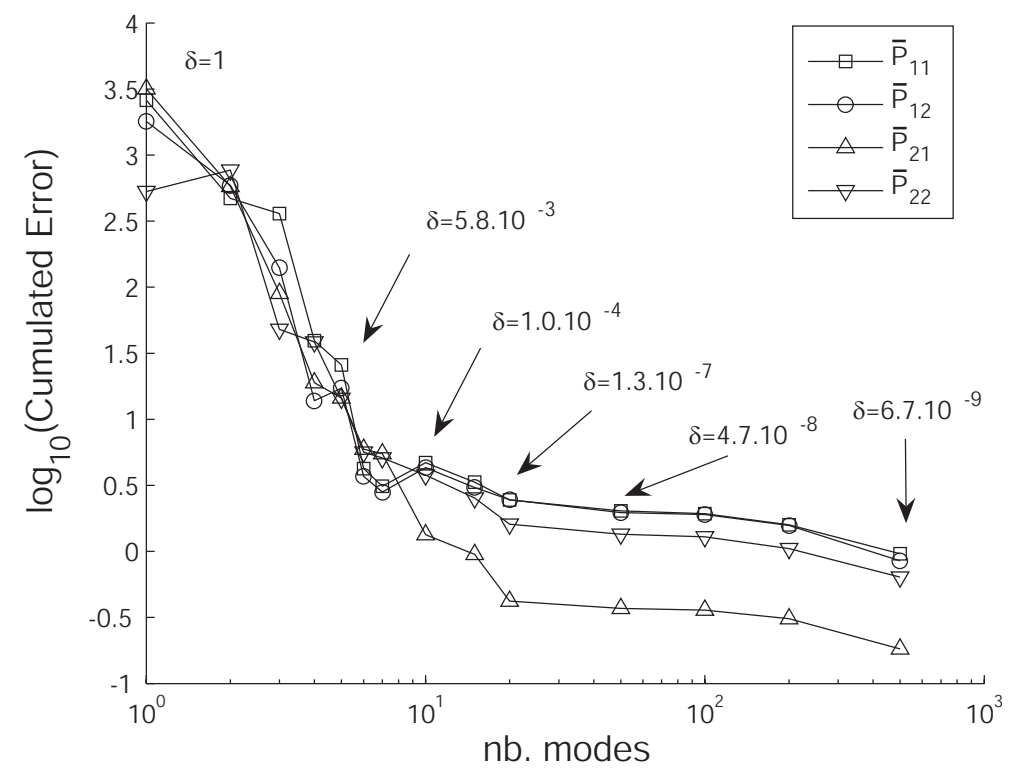

Fig. 13. Convergence of cumulated error versus number of modes and truncature parameter $\delta$.

Table 3. Comparison between full and reduced analysis.

\begin{tabular}{lll}
\hline & Reduced $(20$ dof $)$ & Full (832 dof) \\
\hline $\begin{array}{l}\text { Nb. of iterations until convergence } \\
\text { in each micro problem }\end{array}$ & 2 & 5 \\
\hline $\begin{array}{l}\text { Minimum nb. increment with stable solution } \\
\text { for the whole solution at the macro level }\end{array}$ & 10 & 40 \\
\hline Relative CPU time (whole simulation) & 1 & 16.8 \\
\hline $\begin{array}{l}\text { Size of the database } \\
\text { (history of microproblems) }\end{array}$ & $115 \mathrm{~kb}$ & $4.8 \mathrm{Mb}$ \\
\hline
\end{tabular}

We observe from table 3 and figure 14 that the time computations associated with the global micro/macro analysis are significantly reduced as well as the size of the database. This gain in CPU time is mainly due (a) to the inversion of much smaller matrices in the resolution of the successive nonlinear problems associated with the RVE; (b) the Newton-Raphson algorithm converges faster using the reduced basis, i.e. less iterations are needed to find the solution of the nonlinear micro problems; (c) larger time steps can be used at the macrolevel, which saves a large amount of microproblems to be solved. In this example, only 10 loading increments were necessary to achieve the whole simulation using R3M, against 40 for the full approach. The minimum time steps length was chosen such that no divergence occurs at the microlevel. The time associated with each macro iteration decreases as the solution converges 


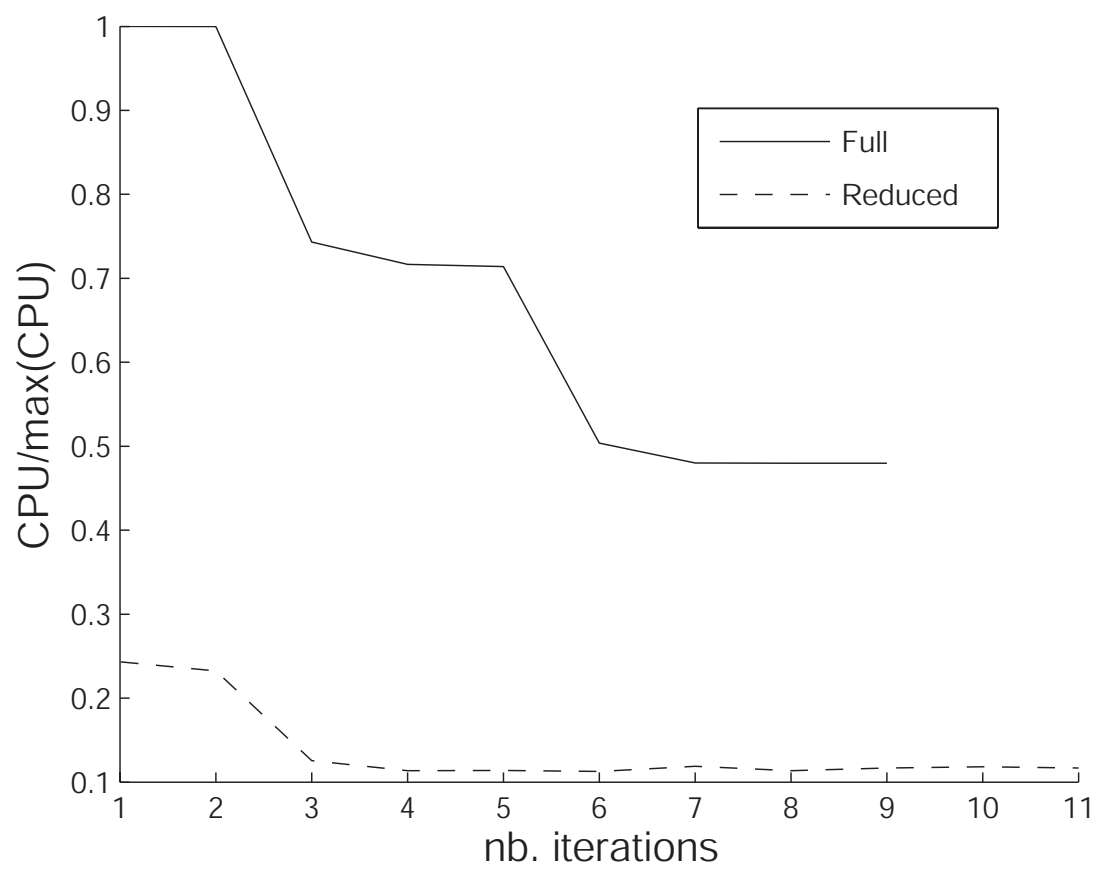

Fig. 14. Time associated with each Newton iteration of the macro problem using full and reduced approaches.

for both approaches, (see figure 14). When the solutions of the microproblems begin to converge, less iterations are involved at the macrolevel.

Finally, we have computed the forces acting on the upper surface $\left(X_{2}=L / 2\right)$ of the macrostructure during the deformations, according to

$$
\mathbf{p}(t)=\int_{\Gamma\left(X_{2}=L\right)} \mathbf{P}(t) \mathbf{N} d \Gamma
$$

The $X_{2^{-}}$component of $\mathbf{p}(t)$ is depicted in figure 15 for the porous hyperelastic material, using full analysis and R3M. Excellent agreement is observed compared to the full solution. As a comparison, the response of an homogeneous material using the Mooney-Rivlin model (20) is also plotted.

\subsection{Compression of elastomer bushing}

An annular bushing composed of a metal inner sleeve, an outer metal sleeve, and an elastomer layer, is subjected to a vertical prescribed displacement as shown in Fig. 16. The annular bushing constitutes the macro structure. Due to the relatively higher stiffness of the metal sleeves, only the elastomer layer is modelled with the outer surface completely fixed and the inner surface moved as a rigid surface in the vertical direction. The elastomer is modelled as a Neo-Hookean hyperelastic material, with properties of Eq. (20) given as 


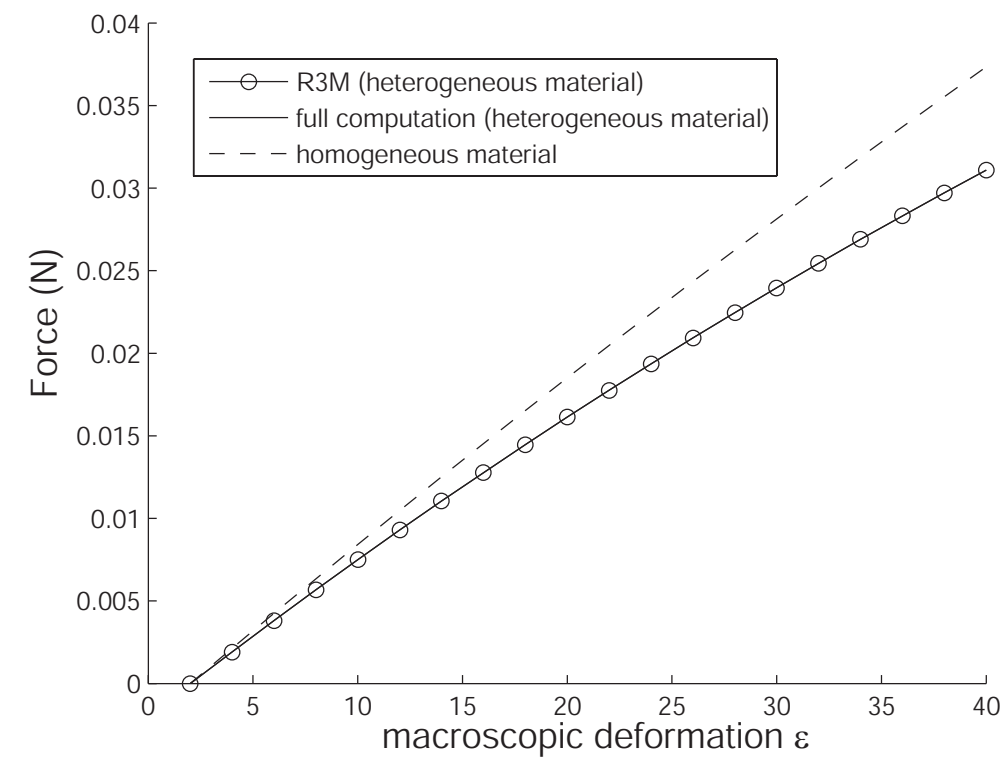

Fig. 15. Resultant force on the upper face of the macro structure; comparison between full and reduced analysis.

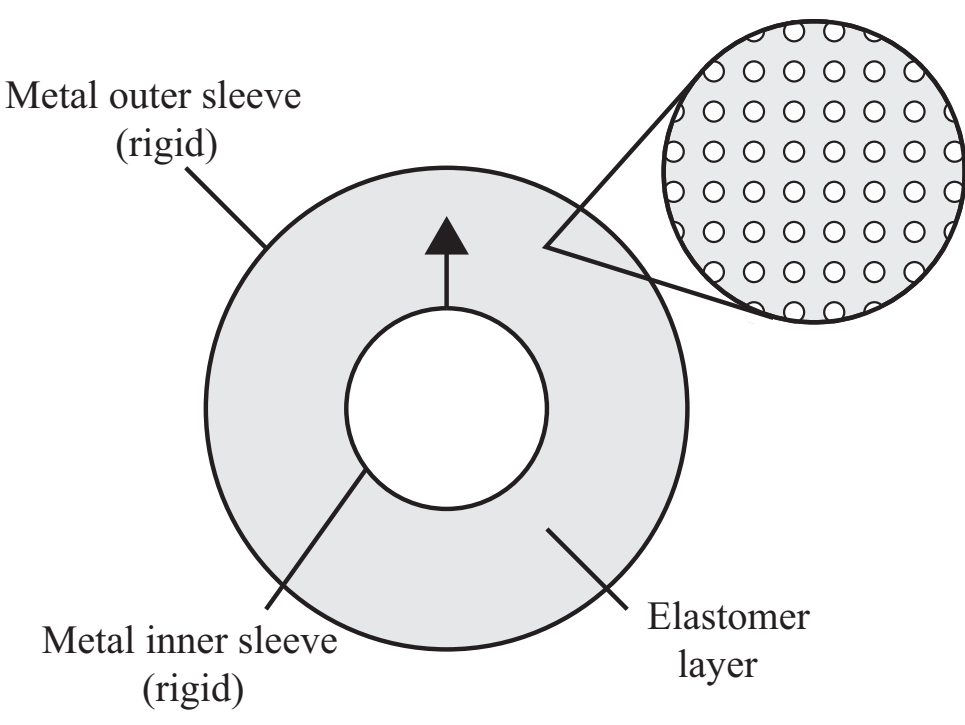

(a)

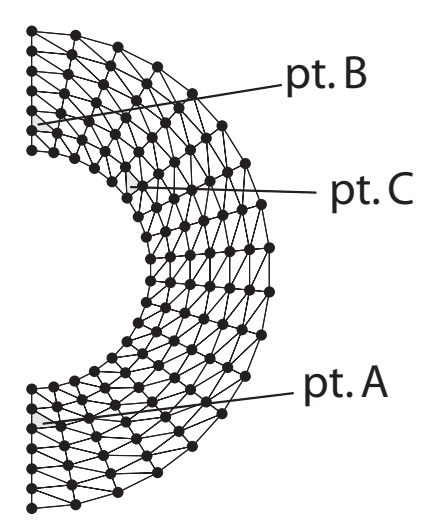

(b)

Fig. 16. Geometry of the elastomer bushing problem.

$c_{1}=6.3 .10^{5} \mathrm{~N} / \mathrm{m}^{2}, c_{2}=0 \mathrm{~N} / \mathrm{m}^{2}$ and $c=100.10^{5} \mathrm{~N} / \mathrm{m}^{2}$. Due to symmetry conditions, only a half of the structure is modelled. The finite element model consists of 126 nodes and a total of 204 integration points.

Here again, we consider porous material, the model of the RVE is identical as in former example. New pre-computations are performed for this new model in order to construct the reduced basis, considering the different parameters of the model, and the values of the stress in the macrostructure. For this prob- 


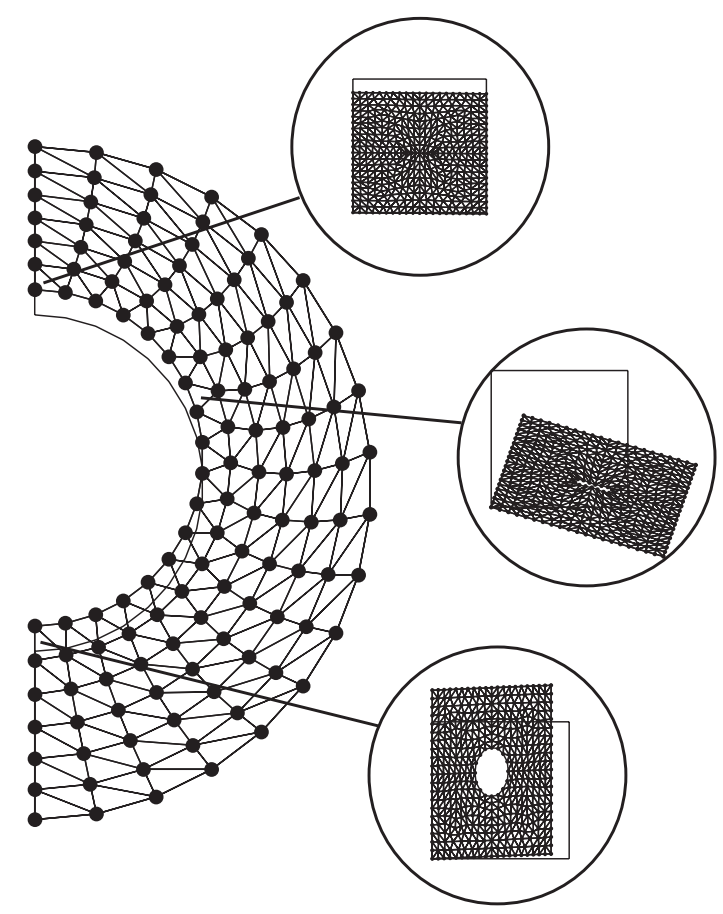

Fig. 17. Deformation of the macro and micro structures.

lem, 29 modes are selected, when $\delta=10^{-7}$ is used. The higher number of basis functions can be explained by the fact that an increase in the material parameter $c$ in (20) induces a higher level of incompressibility, which introduces more geometrical non-linearities, as already noted in the example of section 5.1 .

A multiscale analysis is performed, using a full (reference solution) and reduced approach. The deformation of the macrostructure and some representative microstructures are depicted in figure 17. This model involves larger deformations at the microscale.

In this work, no contact algorithm was implemented in order to take into account the auto-contact of the hole boundaries during compression. We thus have stopped the simulation such as no interpenetration of the hole boundary occurs.

The comparison of homogenized stresses $\bar{P}_{11}, \bar{P}_{12}, \bar{P}_{21}$ and $\bar{P}_{22}$ are depicted in figures 18 (a), 19 (a) and 20 (a). The associated relative errors are depicted in figures 18 (b), 19 (b) and 20 (b).

The Von Mises stress field associated with the porous material in the current configuration is computed according to:

$$
\overline{\boldsymbol{\sigma}}_{y}=\sqrt{\frac{3}{2} \overline{\boldsymbol{\sigma}}_{D}: \overline{\boldsymbol{\sigma}}_{D}}
$$




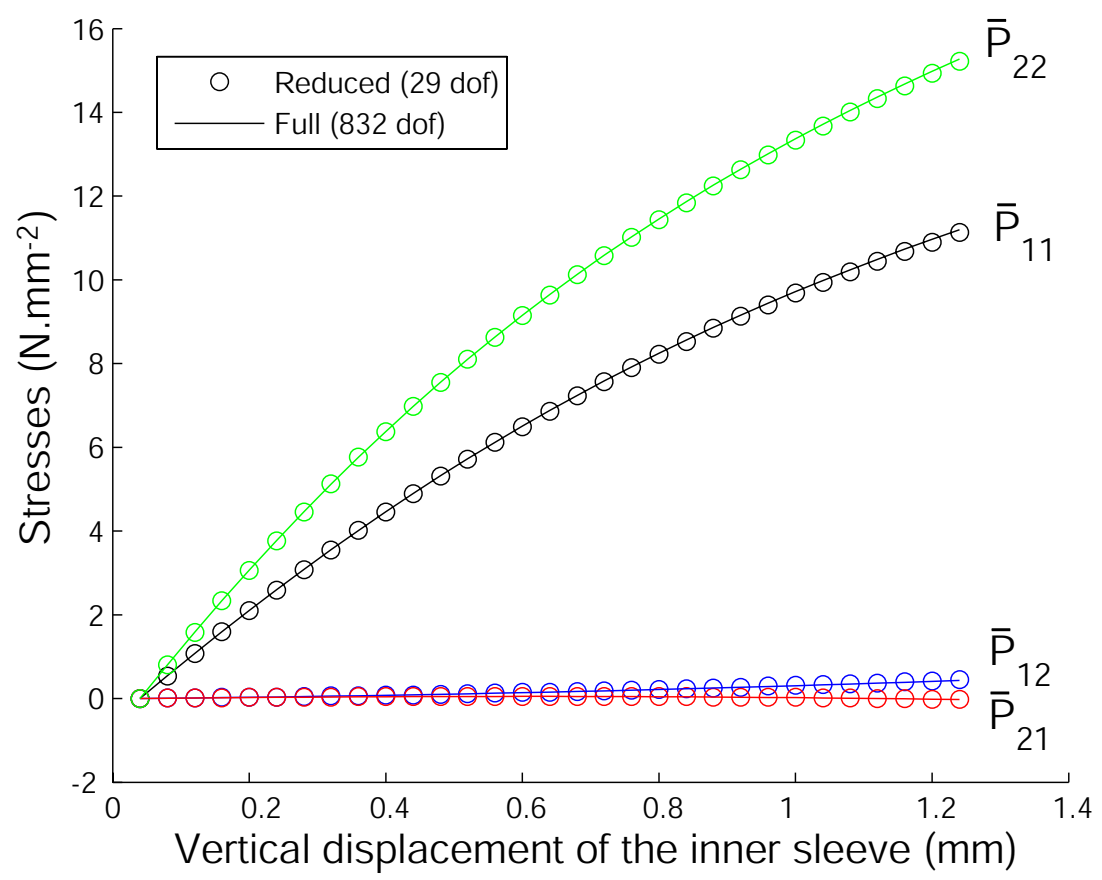

(a)

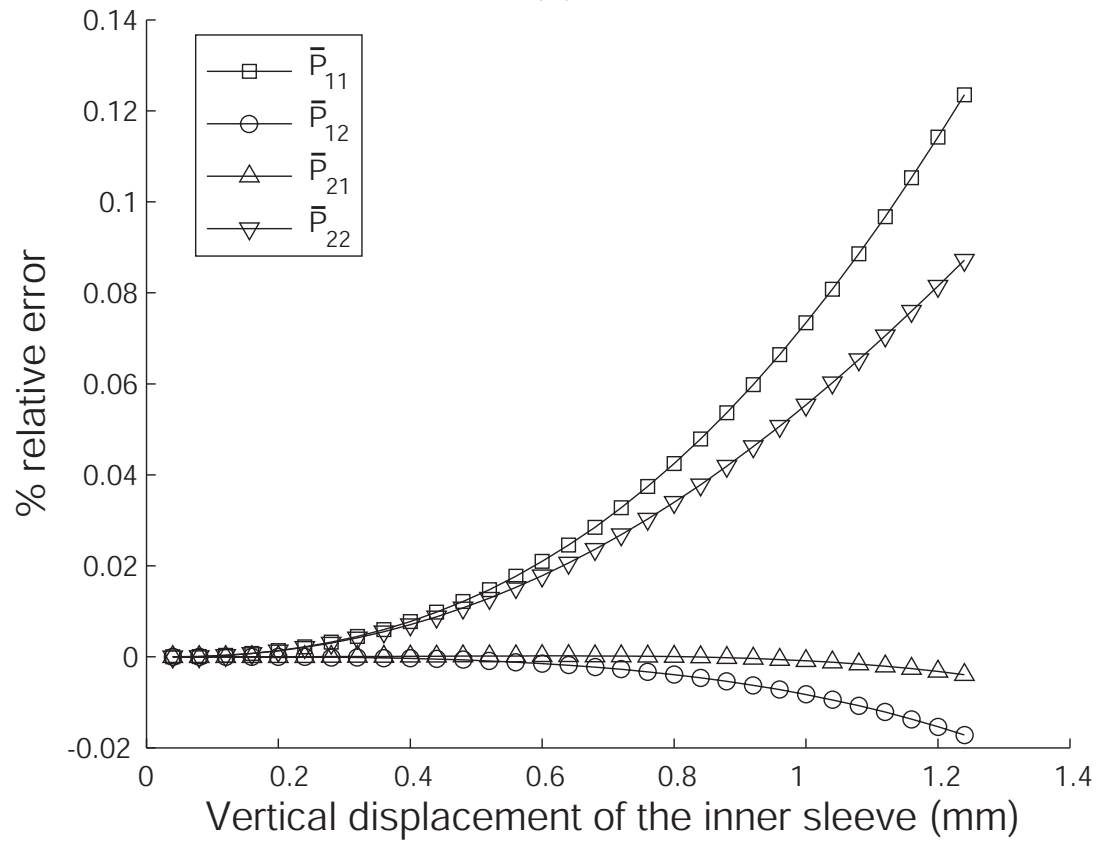

(b)

Fig. 18. Comparison between R3M and full multiscale computation (point A).

where $\overline{\boldsymbol{\sigma}}_{D}$ is the deviatoric part of $\overline{\boldsymbol{\sigma}}, \overline{\boldsymbol{\sigma}}_{D}=\overline{\boldsymbol{\sigma}}-\frac{1}{3} \operatorname{Tr}(\overline{\boldsymbol{\sigma}})$. The associated field representation is depicted in figure 21 .

Good agreement between full and reduced (R3M) approaches is noticed. 


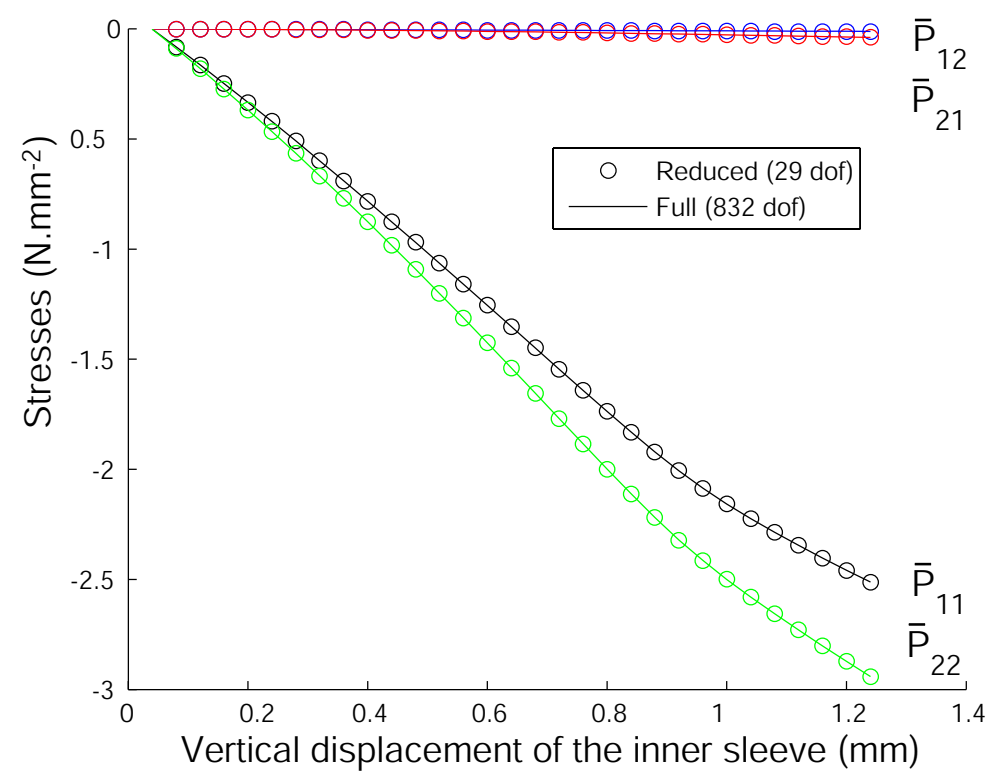

(a)

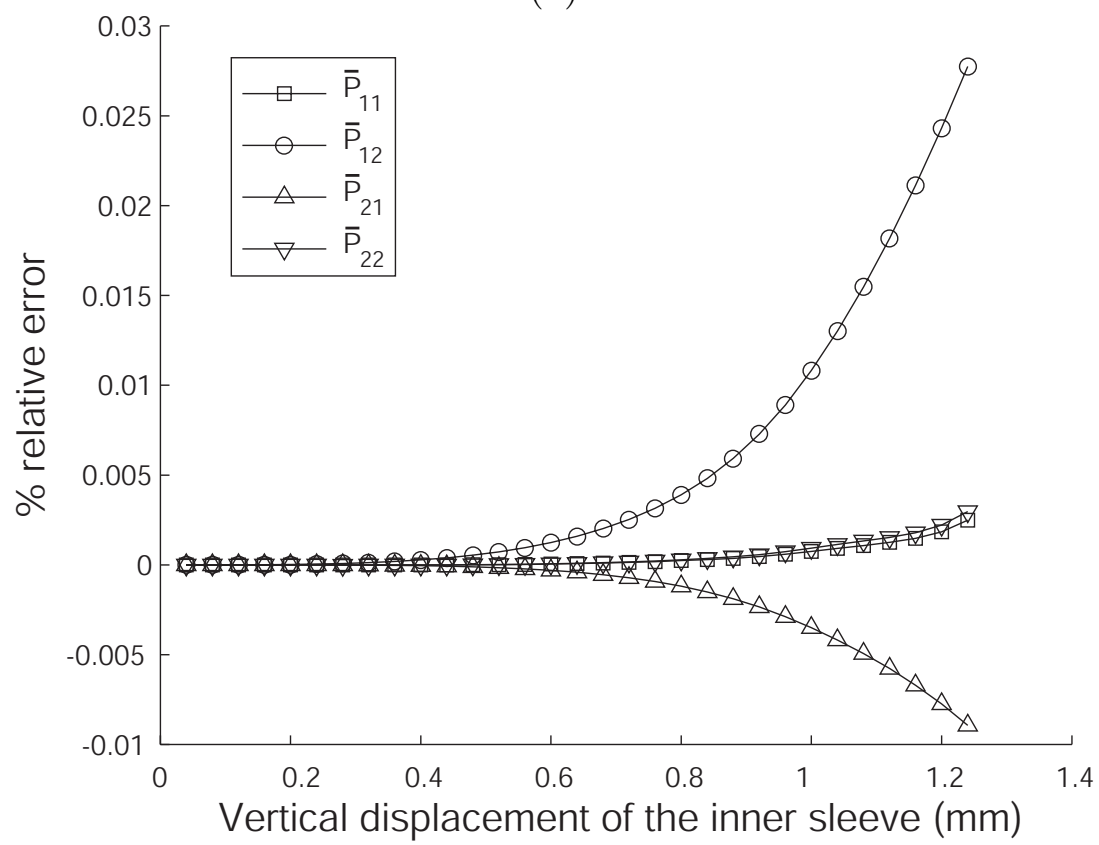

(b)

Fig. 19. Comparison between R3M and full multiscale computation (point B).

\section{Conclusion}

A reduced Model Multiscale Method for the nonlinear homogenization of hyperelastic media in large deformations has been presented. A reduced model procedure has been used to solve the nonlinear problems associated with the microscale. A significant gain in time saving is observed, mainly due to (a) 


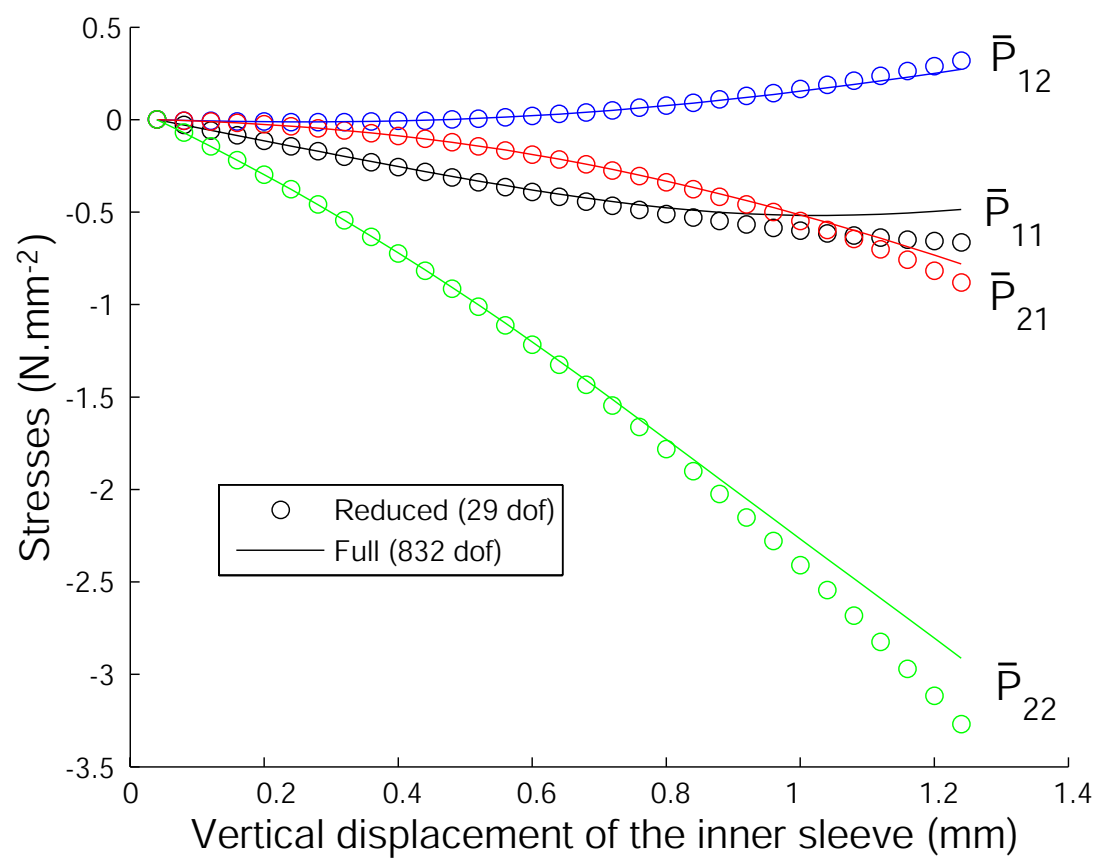

(a)

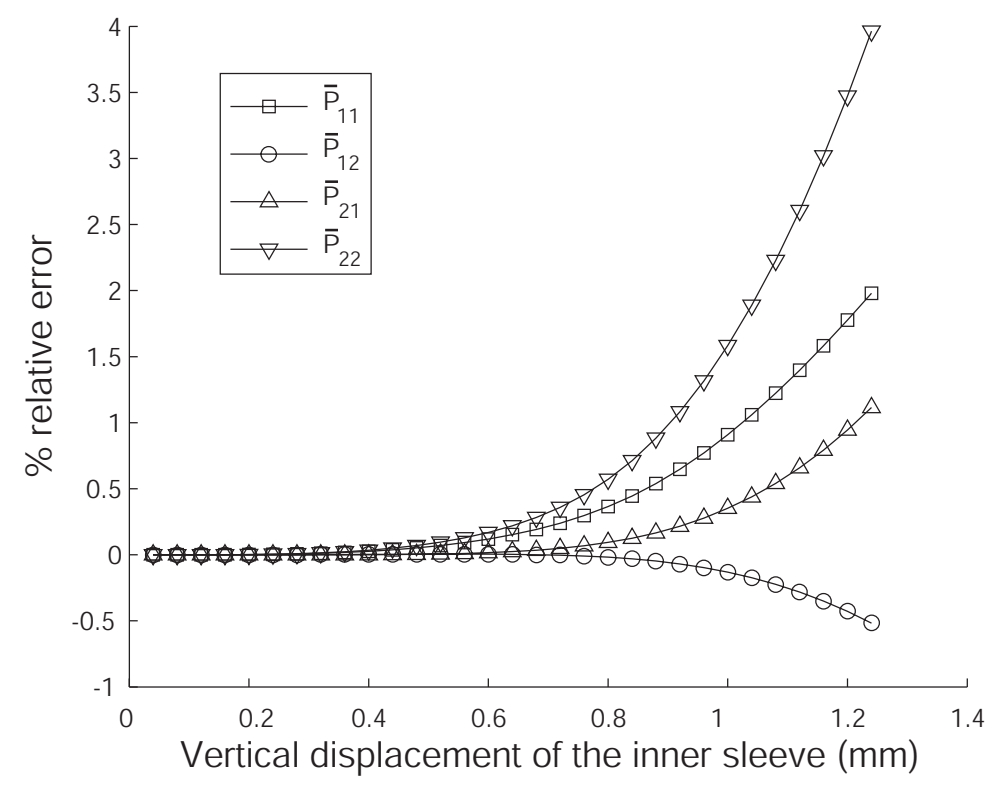

(b)

Fig. 20. Comparison between R3M and full multiscale computation (point C).

only small matrices are inverted; (b) in the context of the Newton-Raphson, the reduced solution of the micro problem converges faster than the full solution; (c) larger time steps (loading increments at the macro level) can be used while maintaining convergence of micro problems, which saves a great amount of nonlinear problems to be solved at the micro level. Moreover, the database describing the evolution of the micro domains is drastically reduced, as the 


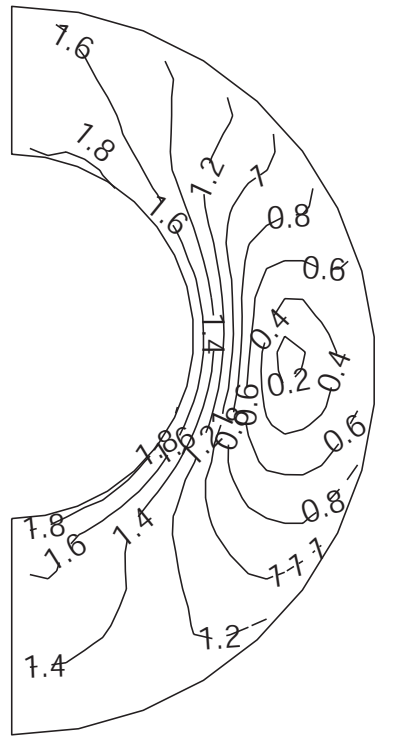

(a)

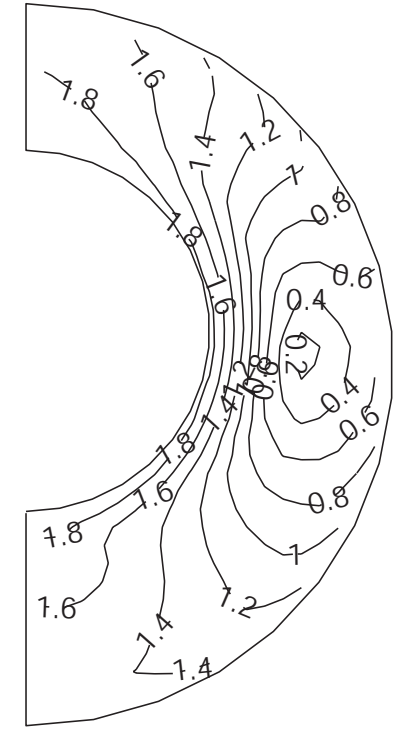

(b)

Fig. 21. Von Mises stresses for full (a) and reduced (b) analysis.

solution can be expressed by a small number of reduced variables.

In this study, the effectiveness of R3M has been examined through porous hyperelastic problems, involving tension, compression and shear at the microlevel at finite strains. A comparison with the full solution, which is used as a reference solution, proves the good accuracy of R3M for different examples with complex loadings at the micro level. A systematic study has been performed to determine the factors affecting the size of the reduced basis. In the present context of hyperelastic media, it seems that the number of selected modes is almost independent of the geometrical complexity as well as the total number of degrees of freedom of the full model. Nevertheless, the size of the reduced basis increases with the geometrical non-linearities of a model (i.e. the level of incompressibility). We have also shown that the choice of the precomputations used to construct the basis is crucial for obtaining an accurate solution.

Possible improvements of the method entails the development of a reduced basis adaptation procedure, in order to handle more sever nonlinearities, more complex boundary conditions, or path-dependent problems. These topics are currently being actively investigated. 


\section{Appendix}

7.1 Stress and elasticity tensor for the Mooney-Rivlin models

The second Piola-Kirchhoff stress tensor is related to $\mathbf{C}$ by:

$$
\mathbf{S}=2 \frac{\partial \Psi(\mathbf{C})}{\partial \mathbf{C}}
$$

Using (20) and (45) we find, after some manipulations:

$$
\mathbf{S}=2\left(c_{1}+c_{2} I_{1}\right) \mathbf{1}-2 c_{2} \mathbf{C}+[2 c J(J-1)-d] \mathbf{C}^{-1}
$$

The first Piola-Kirchhoff stress tensor is related to $\mathbf{S}$ through:

$$
\mathbf{P}=\mathbf{F S}
$$

The fourth-order elasticity tensor in the material description $\mathbf{C}^{e}$ is defined by:

$$
\mathbf{C}^{e}=2 \frac{\partial \mathbf{S}(\mathbf{C})}{\partial \mathbf{C}}=\frac{\partial \mathbf{S}(\mathbf{E})}{\partial \mathbf{E}}=4 \frac{\partial^{2} \Psi}{\partial \mathbf{C} \partial \mathbf{C}}
$$

for the elasticities in the material description, with the major symmetries:

$$
\mathbf{C}^{e}=\mathbf{C}^{e T} \text { or } C_{i j k l}=C_{k l i j}
$$

Using (20) and (48) we obtain the following form of the elasticity tensor $\mathbf{C}^{e}$ for the compressible Mooney-Rivlin model:

$$
\begin{aligned}
& \mathbf{C}^{e}=4 c_{2} \mathbf{1} \otimes \mathbf{1}+2 c J(2 J-1) \mathbf{C}^{-1} \otimes \mathbf{C}^{-1} \\
& -2[2 c J(J-1)-d] \mathbf{C}^{-1} \odot \mathbf{C}^{-1}-4 c_{2} \mathbf{I}
\end{aligned}
$$

with

$$
\left(\mathbf{C}^{-1} \odot \mathbf{C}^{-1}\right)_{i j k l}=\frac{1}{2}\left(C_{i k}^{-1} C_{j l}^{-1}+C_{i l}^{-1} C_{j k}^{-1}\right)
$$

and $\mathbf{I}_{i j k l}=\delta_{i k} \delta_{j l}$. 


\subsection{Matrix forms}

The incremental nonlinear problem requires solving:

$$
\mathbf{K}_{T} \mathbf{\Delta} \mathbf{u}=\mathbf{f}^{e x t}-\mathbf{f}^{\text {int }}
$$

with:

$$
\begin{aligned}
& \mathbf{K}_{T}=\mathbf{K}^{M}+\mathbf{K}^{G} \\
& \mathbf{K}_{I J}^{M}=\int_{\Omega_{0}} \mathbf{B}_{I}^{M} \mathbf{D B}_{J}^{M} d \Omega \\
& \mathbf{K}_{I J}^{G}=\int_{\Omega_{0}} \mathbf{B}_{I}^{F} \mathbf{T} \mathbf{B}_{J}^{F} d \Omega \\
& \mathbf{B}_{I}^{M}=\left[\begin{array}{ll}
F_{11} \frac{\partial N_{I}}{\partial X} & F_{21} \frac{\partial N_{I}}{\partial X} \\
F_{11} \frac{\partial N_{I}}{\partial Y} & F_{21} \frac{\partial N_{I}}{\partial Y} \\
F_{12} \frac{\partial N_{I}}{\partial X} & F_{22} \frac{\partial N_{I}}{\partial X} \\
F_{12} \frac{\partial N_{I}}{\partial Y} & F_{22} \frac{\partial N_{I}}{\partial Y}
\end{array}\right] \quad \mathbf{B}_{I}^{F}=\left[\begin{array}{ll}
\frac{\partial N_{I}}{\partial X} & 0 \\
\frac{\partial N_{I}}{\partial Y} & 0 \\
0 & \frac{\partial N_{I}}{\partial X} \\
0 & \frac{\partial N_{I}}{\partial Y}
\end{array}\right] \\
& \mathbf{T}=\left[\begin{array}{cccc}
S_{11} & S_{12} & 0 & 0 \\
S_{21} & S_{22} & 0 & 0 \\
0 & 0 & S_{11} & S_{12} \\
0 & 0 & S_{21} & S_{22}
\end{array}\right]
\end{aligned}
$$

where $\frac{\partial N_{I}}{\partial X}$ denote the spatial derivatives of the shape functions associated with node $I$.

$$
\begin{aligned}
& \mathbf{D}=\left[\begin{array}{llll}
C_{1111} & C_{1112} & C_{1121} & C_{1122} \\
C_{1211} & C_{1212} & C_{1221} & C_{1222} \\
C_{2111} & C_{2112} & C_{2121} & C_{2122} \\
C_{2211} & C_{2212} & C_{2221} & C_{2222}
\end{array}\right] \\
& \mathbf{f}_{I}^{i n t}=\int_{\Omega_{0}} \mathbf{B}_{I}^{F} \boldsymbol{\Xi} d \Omega, \quad \mathbf{f}_{I}^{e x t}=\int_{\Omega_{0}} \mathbf{N}_{I} \mathbf{B} d \Omega+\int_{\partial \Omega_{0}} \mathbf{N}_{I} \mathbf{t} d \Omega
\end{aligned}
$$


where $\mathbf{N}$ is a matrix containing the shape functions and:

$$
\boldsymbol{\Xi}=\left[\begin{array}{c}
P_{11} \\
P_{12} \\
P_{21} \\
P_{22}
\end{array}\right]
$$

\section{References}

[1] Azeez M.F.A., Vakakis A.F., Numerical and experimental analysis of the nonlinear dynamics due to impacts of a continuous overhung rotor, in: Proceedings of DETC'97, ASME Design Engineering technical Conferences, Sacramento, CA, 1997.

[2] Azeez M.F.A., Vakakis A.F., Proper Orthogonal decomposition (POD) of a class of vibroimpact oscillations. Internal report, Department of Mechanical and Industrial Engineering, University of Illinois at Urbana Champain, U.S.A., 1998.

[3] Chaterjee A., An introduction to the proper orthogonal decomposition, Current Sciences 2000, 78:808-817.

[4] deBotton G., Hariton I., Socolsky E.A. Neo-Hookean fibber reinforced composites in finite elasticity, J. Mech. Phys. Solids. 2006, 54(3):533-559.

[5] Dür A., On the optimality of the discrete Karhunen-Loève expansion, SIAM Journal of Control and Optimization 1998; 36(6):1937-1939.

[6] Dvorak G., Bahei-El-Din Y.A., Wafa A.M., The modeling of inelastic composite materials with the transformation field analysis, Modell. Simulat. Mater. Sci. Engrg. 1994; 2:571-586.

[7] Feeny B.F., Kappagantu R., On the physical interpretation of proper orthogonal modes in vibrations. Journal of Sound and Vibration 1998; 219:189-192.

[8] Feyel F., Chaboche J.-L., FE ${ }^{2}$ multiscale approach for modelling the elastoviscoplastic behaviour of long fibber $\mathrm{SiC} / \mathrm{Ti}$ composite materials, Comput. Methods Appl. Mech. Engrg. 2000; 183:309-330.

[9] Feyel F., A multilevel finite element method $\left(\mathrm{FE}^{2}\right)$ to describe the response of highly non-linear structures using generalized continua, Comput. Methods Appl. Mech. Engrg. 2003; 192:3233-3244.

[10] Feng W., Christensen R.M., Nonlinear deformation of elastomeric foams, Int. J. Non-Linear Mechanics 1982; 17:335-367.

[11] Fish J., Shek K., Pandheeradi M., Shepard M.S., Computational plasticity for composite structures based on mathematical homogenization: theory and practice, Comput. Methods Appl. Mech. Engrg 1997; 148:53-73. 
[12] Gent A.N., Thomas A.G., The deformation of foamed elastic materials, J. Appl. Polym. Sci. 1959; 1:107-113.

[13] Golub G.H., Van Loan C.F., Matrix computations, North Oxford Academic, Oxford, 1983.

[14] Ghosh S., Lee K., Moorthy S., Two scale analysis of heterogeneous elastic-plastic materials with asymptotic homogenization and Voronoi cell finite element model, Comput. Methods Appl. Mech. Engrg 1996; 132:63-116.

[15] Ghosh S., Lee K., Raghavan P., A multi-level computational model for multiscale damage analysis in composite and porous materials, Int. J. Solids Struct. 2001; 38:2335-2385.

[16] Guedes J.M., Kikuchi N., Preprocessing and postprocessing for materials based on the homogenization method with adaptive finite element methods, Comput. Methods Appl. Mech. Engrg 1990; 83:143-198.

[17] Harman H., Modern factor analysis,University of Chicago Press, 1960.

[18] Hashin Z., Large isotropic elastic deformation of composites and porous media, Int. J. Solids Struct. 1985; 21:711-720.

[19] Hill R., On constitutive macro-variables for heterogeneous solids at finite stains, Proc. R. Soc. London Ser. A 1972; 326:131-147.

[20] Hill R., Rice J.R., Elastic potentials and the structure of inelastic constitutive laws, SIAM J. Appl. Math. 1973; 25:448-461.

[21] Hotelling H., Analysis of complex statistical variables in principal components. J. Exp. Psy. 1953; 24:417.

[22] Holmes P., Lumley J.L., Berkooz G., Turbulence, Coherent Structures, Dynamical Systems and Symmetry, Cambridge University Press, Cambridge, 1996;.

[23] Holzapfel G.A., Nonlinear Solid Mechanics, a Continuum Approach for Engineering, Wiley, New-York, 2001.

[24] Humphrey J.D., Cardiovascular Solid Mechanics: Cells, Tissues and Organs, Springer-Verlag, New-York, 2002.

[25] Joyner M.L., Comparison of two techniques for implementing the proper orthogonal decomposition method in damage detection problems, Mathematical and Computer Modelling 2004; 40:553-571.

[26] Karhunen K., Zur spektraltheorie stochastischer prozesse, Ann. Acad. Sci. Fennicae 1946; 37.

[27] Kousnetzova V., Brekelmans W.A.M., Baaijens F.T.P., An approach to micromacro modelling of heterogeneous materials, Computational Mechanics 2001; 27:37-48. 
[28] Kousnetzova V.G., Geers M.G.D., Brekelmans W.A.M., Multi-scale secondorder computational homogenization of multi-phase materials: a nested finite element solution strategy, Comput. Methods Appl. Mech. Engrg 2004; 193:55255550 .

[29] Ladevèze P., Loiseau O., Dureisseix D., A micro-macro and parallel computational strategy for highly heterogeneous structures, Int. J. Numer. Methods Engrg 2001; 52:121-138.

[30] Lall S. Krysl P., Marsden J.E., Structure-preserving model reduction for mechanical systems, Physica D 2003; 184:304-318.

[31] Liang Y.C., Lee H.P., Lim S.P., Lin W.Z., Lee K.H., Proper orthogonal decomposition and its applications-part I:theory. Journal of Sound and vibration 2002; 252(3):527-544.

[32] Lieu T., Lesoinne M., Parameters adaptation of reduced order models for threedimensional flutter analysis, AIAA Papers 2004-0888.

[33] Lieu T., Farhat C., Lesoinne M., Reduced-order fluid/structure modelling of a complete aircraft configuration, Comput. Methods Appl. Mech. Engrg in press.

[34] Loève M.M., Probability Theory, Van Nostrand, N.J., 1955.

[35] Lopez-Pamies O. Ponte Castañeda, Second-order estimated for the macroscopic response and loss of ellipticity in porous rubbers at large deformations, Journal of Elasticity 2005; 76:247-287.

[36] Lorenz E.N., Empirical orthogonal eigenfunctions and statistical weather prediction. Technical report, MIT, Department of Meteorology, 1956. Statistical forecasting project.

[37] Lumley J.L., The structure of inhomogeneous turbulent flows. In A. M. Yaglom and V. I. Tataski, editors, Atmospheric turbulence and radio wave propagation, 1967, pp. 166-178. Nauka, Moscow.

[38] Michel J.C., Moulinec H., Suquet P., Effective properties of composite materials with periodic microstructure: a computational approach, Comput. Methods Appl. Mech. Engrg. 1999; 172:109-143.

[39] Michel J.C., Suquet P., Nonuniform transformation fields analysis, international Journal of Solids and Structures 2003; 40:6937-6955.

[40] Michel J.C., Suquet P., Computational analysis of nonlinear composite structures using the nonuniform transformation fields analysis Comput. Methods Appl. Mech. Engrg. 2004; 193(48-51):5477-5502.

[41] Miehe C., Schröder, Schotte J., Computational homogenization analysis in finite plasticity. Simulation of texture development in polycristalline materials, Comput. Methods Appl. Mech. Engrg. 1999; 171:387-418.

[42] Miehe C., Koch A., Computational micro-to-macro transition of discretized microstructures undergoing small strains, Arch. Appl. Mech. 2002; 72:300-317. 
[43] Miehe C., Computational micro-to-macro transitions for discretized microstructures of heterogeneous materials at finite strains based on the minimization of averaged incremental energy, Comput. Methods Appl. Mech. Engrg. 2003; 192:559-591.

[44] Miehe C., Schotte J., Lambrecht M., Homogenization of inelastic solid materials at finite strains based on incremental minimization principles. Application to the texture analysis of polycristals, Journal of the Mechanics and Physics of Solids 2002; 50:2123-2167.

[45] Müller S., Homogenization of nonconvex integral functionals and cellular elastic materials. Arch. Rational Mech. Anal. 1987; 99:189-212.

[46] Nemat-Nasser S., Hori M., Micromechanics: overall properties of heterogeneous materials. In: North-Holland Series in Applied Mathematics and Mechanics, Vol 37, North-Holland, Amsterdam, 1993.

[47] Newman A., Krishnaprasad P.S., Nonlinear model reduction for RTCVD, in: Proceedings of the 32nd Conference in Information Sciences ans Systems, Princeton, NJ., 1998.

[48] Ogden R.W., On the overall moduli of non-linear elastic composites materials, J. Mech. Phys. Solids 1974; 22:541-553.

[49] Ogden R.W., Extremum principles in non-linear elastic and their application to composites-I, Theory. Int. J. Solids Struct. 1978; 14:265-282.

[50] Pearson K., On lines and planes of closest fit to systems of points in space. Philo Mag. 1901; 6:559.

[51] Ponte Castañeda P., The overall constitutive behavior of nonlinearly elastic composites, Proc. R. Soc. London Ser. A 1989; 422:147-171.

[52] Ponte Castañeda P., Suquet P., Nonlinear composites. Adv. Appl. Mech. 1998; 34:171-302.

[53] Ponte Castañeda P., Second-order homogenization estimates for nonlinear composites incorporating field fluctuations-I. Theory. J. Mech. Phys. Solids 2002; 50:737-757.

[54] Schmidt E., Zur theorie der linearen und nichtlinearen integralgleichungen. I Teil: Etwicklung willkürlicher funktion nach systemen vorgeschriebener. Mathematische Annalen 1907; 63:433-476.

[55] Schmidt R., Glauser M., Improvements in low dimensional tools for flowstructure interaction problems: using global POD, AIAA Papers 2004-0889.

[56] Sheng N., Boyce M.C., Parks D.M., Rutledge G.C., Abes J.I., Cohen R.E. Multiscale micromechanical modelling of polymer/clay nanocomposites and their effective clay particle. Polymer 2004; 45:487-506.

[57] Smit R.J.M., Brekelmans, Meijer H.E.H., Prediction of the mechnanical behaviour of non-linear heterogeneous systems by multi-level finite element modeling, Comput. Methods Appl. Mech. Engrg 1998; 155:181-192. 
[58] Suquet P.M., Local and global aspects in the mathematical theory of plasticity, in: A. Sawczuck, G. Bianchi (Eds.), Plasticity Today: Modelling, Methods and Applications, Elsevier Applied Science Publishers, London, 1985, pp. 279-310.

[59] Suquet P.M., Elements of homogenization for inleastic solid mechanics. In: Sanchez-Palenzia E. Zaoui A. (Eds), Homogenization Techniques for composites Materials, Lecture Notes in Physics, Vol. 272, Springer, Berlin, 1987, pp. 193278.

[60] Tatyor J., Dynamics of large scale structures in turbulent shear layers, PhD. thesis, Clarkson University, 2001.

[61] Terada K., Kikuchi N., Nonlinear homogenization method for practical applications, in: S. Ghosh, M. Ostoja-Starzewski (Eds.), Computational Methods in Micromechanics Plasticity Today: Modeling, Methods and Applications, London, Elsevier Applied Science Publishers, 1985, pp. 279-310.

[62] Terada K., Kikuchi, A class of general algorithms for multi-scale analysis of heterogeneous media, Comput. Methods Appl. Mech. Engrg 2001; 190:5427-5464.

[63] Willis J.R., Variational and related methods for the overall properties of composites. in: Advances in Applied Mechanics, Vol 21, Academic Press, New York, 1981, pp. 1-78. 